\title{
A solution to temporal credit assignment using cell-type-specific modulatory signals
}

\author{
Yuhan Helena Liu ${ }^{1, *}$, Stephen $\mathrm{Smith}^{2}$, Stefan Mihalas ${ }^{2}$, Eric Shea-Brown ${ }^{1,2,3}$, and Uygar Sümbül, ${ }^{2 *}$ \\ ${ }^{1}$ Department of Applied Mathematics, University of Washington, Seattle, WA, USA \\ ${ }^{2}$ Allen Institute, 615 Westlake Ave N, Seattle WA, USA \\ ${ }^{3}$ Computational Neuroscience Center, University of Washington, Seattle, WA, USA \\ ${ }^{*}$ Correspondence: hyliu24@uw.edu, uygars@alleninstitute.org
}

\begin{abstract}
Animals learn and form memories by jointly adjusting the efficacy of their synapses. How they efficiently solve the underlying temporal credit assignment problem remains elusive. Here, we re-analyze the mathematical basis of gradient descent learning in recurrent spiking neural networks (RSNNs) in light of the recent single-cell transcriptomic evidence for cell-type-specific local neuropeptide signaling in the cortex. Our normative theory posits an important role for the notion of neuronal cell types and local diffusive communication by enabling biologically plausible and efficient weight update. While obeying fundamental biological constraints, including separating excitatory vs inhibitory cell types and observing connection sparsity, we trained RSNNs for temporal credit assignment tasks spanning seconds and observed that the inclusion of local modulatory signaling improved learning efficiency. Our learning rule puts forth a novel form of interaction between modulatory signals and synaptic transmission. Moreover, it suggests a computationally efficient learning method for bio-inspired artificial intelligence.
\end{abstract}

\section{Introduction}

Animals learn and form memories by adjusting the efficacy of particular subsets of the myriad synaptic connections that establish their nervous system architectures. Borrowing terminology that was introduced in the early days of artificial intelligence [1], identification of the connection subset necessary for adaptive learning has come to be known by neuroscientists, too, as "credit assignment" - that is, the assignment of credit (or blame) to particular synaptic connections as needed to guide the strengthening (or weakening) to achieve adaptive memory formation. Unfortunately, the mechanisms of credit assignment for biological networks remain deeply enigmatic [2, 3, 4].

Mathematical "gradient backpropagation" algorithms [5, 6] now solve the problem of credit assignment for artificial neural networks sufficiently well to have ushered in an era of shockingly powerful artificial intelligence, but the training of networks by these algorithms still face oppressive scalability issues. Computer scientists therefore continue to look to neuroscience for inspiration regarding new approaches to credit assignment. Neuroscientists meanwhile still struggle to see how the computer scientists' backpropagation approach to credit assignment could be implemented by the brain's "hardware". Inspired by new findings regarding neuromodulatory signaling from single-cell RNA sequencing analysis of mouse brain gene expression [7, 8], we have developed and propose here a simple theory which may contribute to a new wave of progress in understanding biological credit assignment and may also serve to inspire more efficient credit assignment in the artificial intelligence realm.

One conspicuous gap between computational models of neuronal networks and experimental data appears in the concept of cell types. Recently, remarkable diversity and stereotypy have been observed in neuronal phenotypes 19, 10, 11, 12, 13. Despite attempts at bridging this gap by explicitly studying discrete cell types as part of the computational model [14], an overarching role for cell types in synaptic plasticity is not yet known.

Hebbian plasticity, a local learning rule that prescribes lasting change in synaptic strength based on correlations of spike timing between particular presynaptic and postsynaptic neurons, has long been recognized as one biologically plausible basis for assigning credit to particular synapses during memory formation [15, 16]. Both experimental and theoretical investigations now indicate forcefully, however, that a Hebbian rule alone is insufficient: impacts of correlated spike timing must be augmented by one or more additional, modulatory factors $17,18,19,20,2,21,22$, 


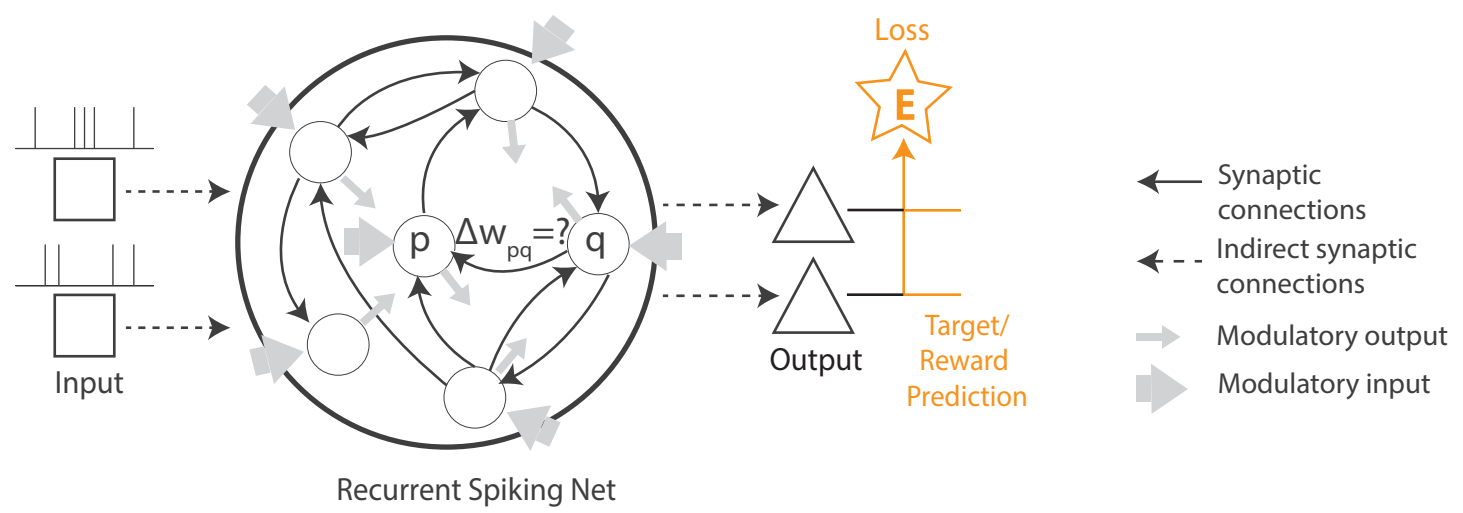

Figure 1: Temporal credit assignment through the interplay of synaptic transmission and modulatory signaling. The network is tasked with producing a desired output signal given a certain input. The challenge involves determining how much each weight (out of potentially thousands or millions of connections) is responsible for overall network performance, so as to guide the strengthening and weakening of the individual weights. In this view of the neuronal network as a stack of synaptic and modulatory networks, learning (i.e the update of synaptic weights $w$ ) is shaped by both local synaptic activity and modulatory signaling.

$23,24,25,26,27$. Dopamine, a neuromodulatory monoamine secreted by axons ramifying widely from midbrain in response to salient "rewarding" or "surprising" events, has emerged as the most prominent candidate for such a factor 28 . Neurons respond to secreted dopamine strictly via activation of G protein-coupled receptors (GPCRs), which act via intracellular messengers upon ion channels, secretory machinery and gene expression over widely varied time scales to regulate membrane excitability, synaptic strength and the temporal parameters of Hebbian plasticity rules.

Five different genes encoding dopamine-selective GPCRs are expressed in highly differential, cell-type-specific fashion by central nervous system (CNS) neurons, but so are hundreds of other genes encoding GPCRs of widely varying ligand selectivity [29]. A majority of these other GPCRs are selective for diffusible ligands that are secreted in response to neuronal activity and many are recognized, like dopamine, as potent neuromodulators [30, 31, 32, 33. These neuromodulatory GPCRs include receptors selective for other monoamines such as serotonin, norepinephrine and histamine, metabotropic receptors for the neurotransmitters glutamate and GABA, the muscarinic acetylcholine receptors, endocannabinoid receptors, and receptors selective for neuropeptides 34]. Most of these other GPCRs have downstream signaling actions quite similar to those of the dopamine-selective GPCRs and many furthermore have been shown to impact memory across a wide range of animal species [35, 36, 37]. Thus, numerous neuromodulatory GPCRs beyond the dopamine receptors merit serious consideration as potential additional factors contributing to credit assignment. Only a few are likely to act like dopamine as top-down reward signals, but many are likely to distribute information about local network activity locally in ways that might contribute significantly to the speed and efficiency of credit assignment.

The present work was inspired especially by new single-cell RNA sequencing (scRNA-seq) evidence for surprisingly abundant and diverse expression of neuropeptide precursor protein (NPP) and NP-selective GPCR (NP-GPCR) genes in virtually all mouse neocortical neurons [7, 8]. Active neuropeptide (NP) molecules are proteolytic fragments of proteins encoded by NPP genes and released from neurons in activity-dependent fashion. NPs comprise the largest family of diffusible ligands for neuromodulatory GPCRs $[38,39$ and many experimental findings have implicated them in memory storage processes $37,40,41$. The fundamental importance of NP signaling is supported by their extremely deep conservation across all animal species [42] and by extremely high levels of NPP-encoding mRNA abundance in individual neurons 7 . The most widely known NPs are the opioids (enkephalins, endorphins and others) and the pro-social peptide oxytocin, but there are many others [43. In mammalian CNS, products of approximately $50 \mathrm{NPP}$ genes act via NP-GPCR proteins encoded by approximately 80 different NP-GPCR genes. The scRNA-seq data further show that almost all mouse CNS neurons express several NPPs and several NP-GPCRs and that, for both families, the combinations expressed are extraordinarily cell-type-specific [7]. Though secreted NP molecules must diffuse far enough through brain interstitial spaces to reach many individual synapses connecting neurons representing multiple types [44, 45], tightly defined patterns of sparse, cell-type-specific expression of particular signal source (NPP) and signal receiver (NP-GPCR) genes nonetheless arguably define directed graphs of connectivity between nearby neurons of particular types. These findings thus suggest a "multidigraph" view of cortical computation and plasticity, founded upon interplay between fast synaptic and slow neuromodulatory 
networks, definable by multiple directed graphs sharing cell types as common nodes [8] (Figure 1). Here we explore this prospect theoretically in the hope of motivating and facilitating experimentation and developing experimentally testable predictions.

A major step forward in biologically plausible learning in recurrent neural networks (RNNs) - widely-adopted high dimensional dynamical models of neural circuits for robust performance in temporal tasks [46] - was brought by two recent theoretical studies that developed local and causal learning rules [47, 48]. These studies derived local approximations to gradient-based learning in RNNs by requiring synaptic weight updates to depend only on local information about pre- and postsynaptic activities in addition to a top-down learning signal pertaining to network output error. While Murray approximated RTRL for rate-based networks [47, Bellec et al. approximated BPTT to train recurrent spiking neural networks (RSNNs) [48]; this spike-based communication yields greater biological plausibility and energy efficiency $44,50,51,52,53$.

Building on these recent advances, we test the plausibility of the abstract multidigraph concept by formulating it into an explicit computational model and describe a computational role for cell types in synaptic learning as part of our model. More specifically, we truncate the RTRL algorithm to remove nonlocal dependencies, but include modulatory terms respecting neuronal types to provide nonlocal information in the form of diffusive signaling. (truncations of the RTRL algorithm have received recent attention from the machine learning and neuromorphic hardware communities [54, 55].) Our multidigraph learning rule (MDGL) generalizes multi-factor learning, in which a Hebbian component is combined with local cell-type-specific modulatory signals in addition to the top-down instructive signals. We train the multiple-cell-type RSNNs with MDGL to perform tasks involving temporal credit assignment over a timescale of seconds. Although we focus on supervised learning, our theory can be extended to reinforcement learning settings 48. Our proof-of-concept implementation of MDGL shows significant improvements over previous literature and advances the field of biologically plausible temporal credit assignment. From a neuroscience perspective, our study proposes a new model of cortical learning shaped by the interplay of local modulatory signaling and synaptic transmission, and potentially brings us closer to understanding biological intelligence. From a computer science perspective, our method offers an energy efficient method for on-chip neuro-inspired AI.

\section{Results}

\section{Mathematical basis of multidigraph learning in RSNNs}

To study the basic principles governing plasticity in neuronal circuits, we use a simple and widely adopted recurrent neural network model with the addition that we endow neurons with local modulatory signaling and cell type-specific cognate reception (Figure 1). An overview of our biologically plausible learning framework along with other methods investigated in this work are summarized in Figure 2.

The RSNN used in this study (Figures 1 and 3 a ) takes $N_{i n}$ spiking inputs $x_{i, t}$ for $i=1,2, \ldots N_{\text {in }}$ and $t=1,2, \ldots, T$, where $x_{i, t}$ assumes the value 1 if input unit $i$ fires at time $t$ and 0 otherwise. These inputs are sent to the spiking recurrent units. Internal to recurrent unit $j$ is state $s_{j, t}$, which denotes the internal state (e.g. membrane potential for LIF neurons) of cell $j$ at time $t$. The output of the $j^{\text {th }}$ recurrent neuron at time $t, z_{j, t}$ for $j=1,2, \ldots N$, also takes the value 1 if recurrent neuron $j$ fires at time $t$ and 0 otherwise. The recurrent activity is read out to graded output $y_{k, t}$ for $k=1,2, \ldots N_{y}$, whose performance for a given task incurs a feedback signal $E$ (e.g., error or negative reward). Throughout, many variables of interest display temporal dependencies and spatial (e.g., cell index $p$, synapse index $p q$ for connections from presynaptic neuron $q$ to postsynaptic neuron $p$ ) dependencies. Equations governing the dynamics of the non-adaptive and adaptive leaky integrate-and fire (LIF) neurons used in this paper are given in Methods.

We study iterative adjustment of all synaptic weights (input weights $w^{I N}$, recurrent weights $w$ and output weights $w^{O U T}$ ) using gradient descent on $E$, which involves a weight update in the direction of error gradient (see Methods for gradient descent and detailed definitions of $E$ ). This error gradient can be calculated with BPTT or RTRL by unwrapping the RSNN dynamics over time; this unwrapping is needed because weights influence past network activity, which then influences present and future activity through Eq. M1. Methods (Figure 3p). While these two algorithms yield equivalent results, their bookkeeping for the gradient calculations differs [4]. Gradient calculations in BPTT depend on future activity, which poses an obstacle for online learning and biological plausibility. Unlike BPTT, the computational dependency graph of RTRL is causal. Therefore, we focus our analysis on RTRL and factor the error gradient across time and space (Eqs. M8 and M9, Methods).

Key problems that RTRL poses to biological plausibility and computational cost reside in the factor $\frac{\mathrm{d} s_{j, t}}{\mathrm{~d} w_{p q}}$ that 
ai

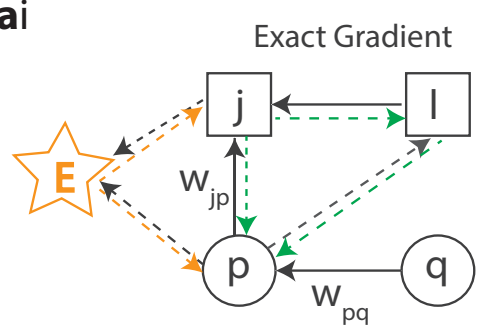

iii

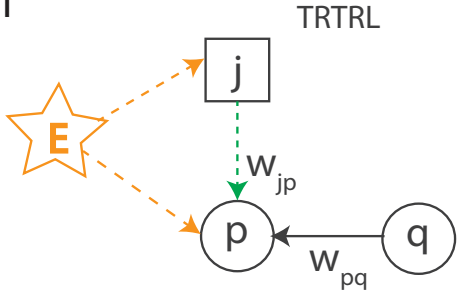

ii

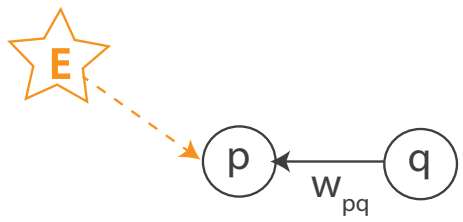

iv

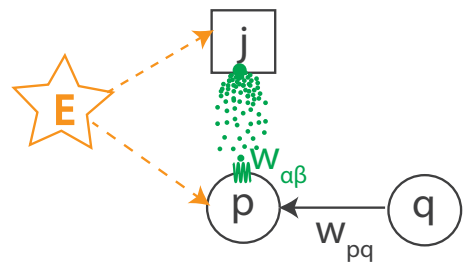

v

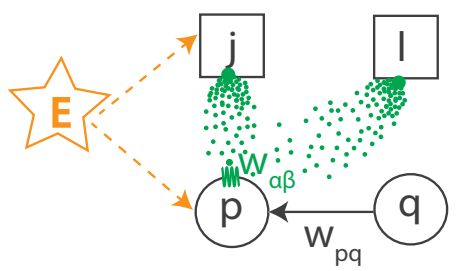

b
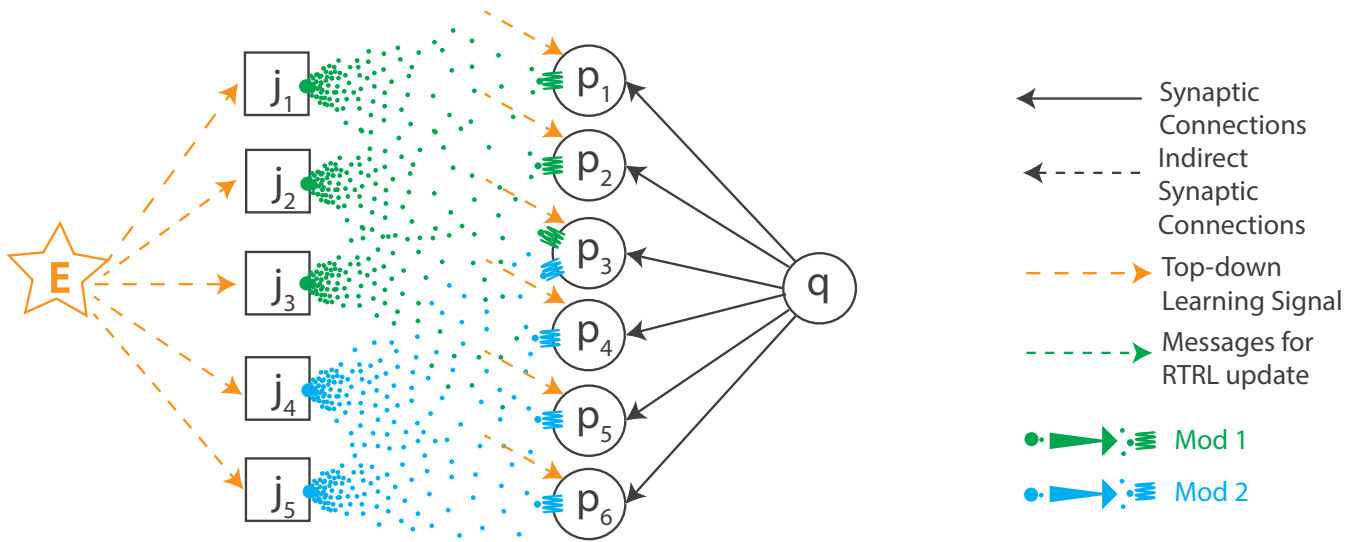

Cell-type-specific-signaling in MDGL

Figure 2: Biologically plausible temporal credit assignment using cell-type-specific modulatory signals. a) Learning rules investigated include (i) the exact gradient: updating weight $w_{p q}$, the synaptic connection strength from presynaptic neuron $q$ to postsynaptic neuron $p$, involves nonlocal information inaccessible to neural circuits, i.e. the knowledge of activity for all neurons $j$ and $l$ in the network. This is because $w_{p q}$ affects the activities of many other cells through indirect connections, which will then affect the network output at subsequent time steps. As in Figure 1, E quantifies the network's performance. (ii) E-prop, a state-of-the-art local learning rule, restricts weight update to depend only on pre- and post-synaptic activity as well as a top-down learning signal. (iii) Our truncated weight update (TRTRL) includes dependencies within one connection step, which are omitted in e-prop. (iv) Our multi-digraph learning rule (MDGL) incorporates local cell-type-specific modulatory signaling through which the activity of neuron $\mathrm{j}$ can be delivered to neuron p. (v) NL-MDGL: A nonlocal version of MDGL, where modulatory signal diffuses to all cells in the network. b) Illustration of cell-type-specific modulatory signaling in MDGL: source neurons $j_{1}, j_{2}$ and $j_{3}$ express the same precursor type, and likewise for $j_{4}$ and $j_{5}$. Neurons $p_{1}$ to $p_{6}$ can be grouped into three types based on the different combination of receptors they express. This results in a modulatory network described by a cell-type-specific channel gain on top of the classical synaptic network.

arises during the factorization of the gradient (Eq. M8 and Eq. M9. The factor $\frac{\mathrm{d} s_{j, t}}{\mathrm{~d} w_{p q}}$ keeps track of all direct and indirect dependencies of neuron state $j$ on weight $w_{p q}$. In other words, this factor accounts for both the spatial and temporal dependencies in RSNNs: state dependencies across time $t$, as explained above, result from unwrapping the temporal dependencies illustrated in Figure 3b; state dependencies across space, however, are due to the indirect dependencies (of all $z_{t}$ on $w$ and all $z_{t^{\prime}}\left(t^{\prime}<t\right)$ ) arising from recurrent connections (Figure 3c). These recurrent dependencies are all accounted for in the $\frac{\mathrm{d} s_{j, t}}{\mathrm{~d} w_{p q}}$ factor, which can be obtained recursively as follows:

$$
\frac{\mathrm{d} s_{j, t}}{\mathrm{~d} w_{p q}}=\frac{\partial s_{j, t}}{\partial w_{p q}}+\sum_{l} \frac{\partial s_{j, t}}{\partial s_{l, t-1}} \frac{\mathrm{d} s_{l, t-1}}{\mathrm{~d} w_{p q}}
$$


bioRxiv preprint doi: https://doi.org/10.1101/2020.11.22.393504; this version posted March 6, 2021. The copyright holder for this preprint (which was not certified by peer review) is the author/funder, who has granted bioRxiv a license to display the preprint in perpetuity. It is made available under aCC-BY-NC 4.0 International license.

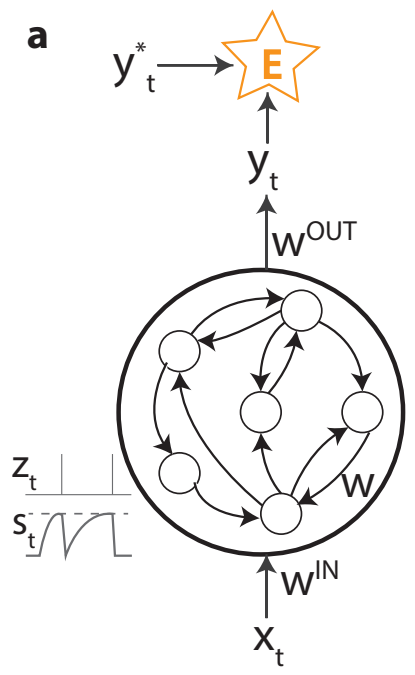

b

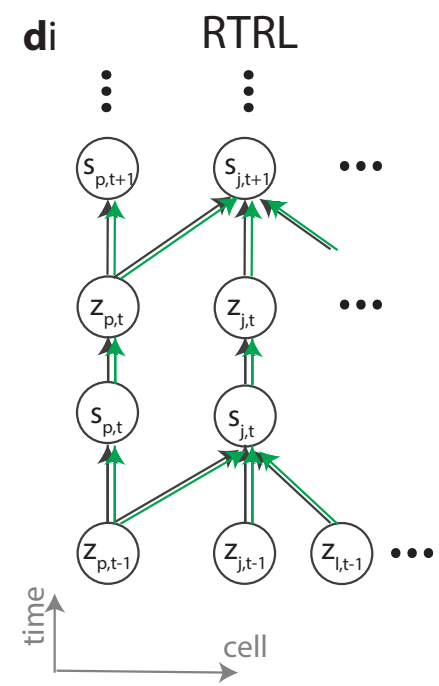

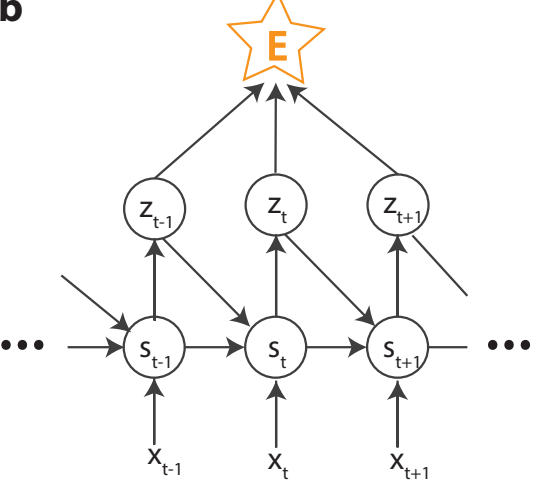

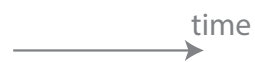

dii

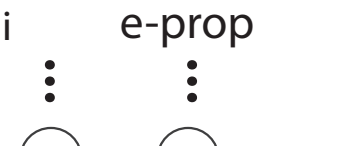

c
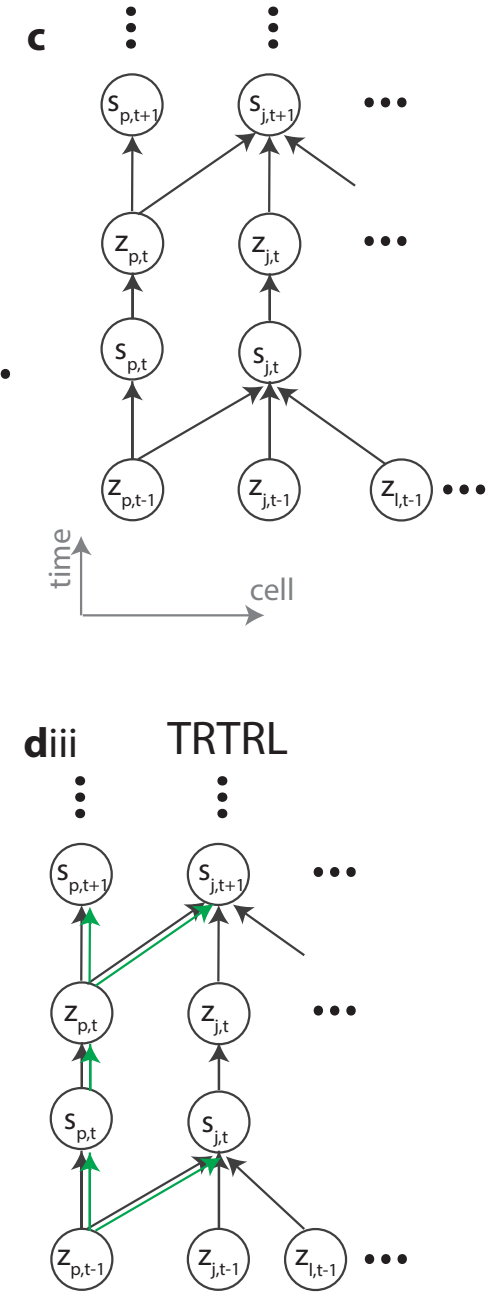
remains. This gap is not surprising given that both algorithms account only for pre- and post-synaptic activities, ignoring - by design - the many potential contributions to optimal credit assignment from neurons that do not participate directly in the synapse of interest.

\section{A potential role for cell type-specific modulatory signals}

To reveal a potential role for cell-type-based modulatory signals in synaptic plasticity, we begin by partially restoring non-local dependencies between cells - those within one connection step. This is the truncated RTRL framework (Figures 3diii, 2 aiii), and the memory trace term $\frac{\mathrm{d} s_{j, t}}{\mathrm{~d} w_{p q}}$ becomes

$$
\frac{\mathrm{d} s_{j, t}}{\mathrm{~d} w_{p q}} \approx \begin{cases}\frac{\partial s_{j, t}}{\partial z_{p, t-1}} \frac{\partial z_{p, t-1}}{\partial s_{p, t-1}} \frac{\mathrm{d} s_{p, t-1}}{\mathrm{~d} w_{p q}}=w_{j p} \frac{\partial z_{p, t-1}}{\partial s_{p, t-1}} \frac{\mathrm{d} s_{p, t-1}}{\mathrm{~d} w_{p q}}, & p \neq j \\ \frac{\partial s_{j, t}}{\partial w_{j q}}+\frac{\partial s_{j, t}}{\partial s_{j, t-1}} \frac{\mathrm{d} s_{j, t-1}}{\mathrm{~d} w_{j q}}, & p=j\end{cases}
$$

Thus, when $j=p$, our truncation implements $\frac{\mathrm{d} s_{p, t}}{\mathrm{~d} w_{p q}} \approx \frac{\partial s_{j, t}}{\partial w_{j q}}+\frac{\partial s_{j, t}}{\partial s_{j, t-1}} \frac{\mathrm{d} s_{j, t-1}}{\mathrm{~d} w_{j q}}$, which coincides with e-prop. Eq. 2 adds the case when $p \neq j$, for which $\frac{\mathrm{d} s_{j, t}}{\mathrm{~d} w_{p q}}$ was simply set to 0 in e-prop. We note that the truncation in Eq. 2 resembles the n-step RTRL approximation recently proposed in [54, known as SnAP-n, which stores $\frac{\mathrm{d} s_{j, t}}{\mathrm{~d} w_{p q}}$ only for $j$ such that parameter $w_{p q}$ influences the activity of unit $j$ within $n$ time steps. The computations of SnAp-n converge to those of RTRL as n increases. Our truncation in Eq. 2 is similar to SnAp-n with $n=2$ with two differences: (i) we apply it to spiking neural networks, (ii) we drop the previous time step's Jacobian term $\frac{\mathrm{d} s_{j, t-1}}{\mathrm{~d} w_{p q}}$, which would necessitate the maintenance of a rank-three ("3-d") tensor with costly storage demands $\left(O\left(N^{3}\right)\right)$ and for which no known biological mechanisms exist. Thus, the truncation in Eq. 2 requires the maintenance of only a rank-two ("2-d") tensor specific to synapse $p q$, which can be realized via an eligibility trace as we explain next.

By substituting equation Eq. 2 into Eq. M8 and Eq. M9, we approximate the overall gradient as

$$
\frac{\widehat{\mathrm{d} E}}{\mathrm{~d} w_{p q}}=\left.\sum_{t} \frac{\mathrm{d} E}{\mathrm{~d} w_{p q}}\right|_{\mathrm{e}-\text { prop }}+\underbrace{\sum_{j} a_{j, t} w_{j p} e_{p q, t-1}}_{:=\widehat{\Gamma_{p q, t}}},
$$

where $a_{j, t}$ (Eq. M12 denotes the activity-dependent modulatory signal emitted by neuron $j$ at time $t$ and $e_{p q, t}$ (Eq. M13) is the eligibility trace maintained by postsynaptic cell $p$ to keep a memory of the preceding activity of presynaptic cell $q$ and postsynaptic cell $p$ (Methods). There have been numerous suggestions that some persistent "eligibility trace" must exist to bridge the temporal gap between correlated firing at specific synapses and laterarriving, dopamine-like reward signals $[21,26,56,57,58,59]$. In Eq. 3, the first term alone gives exactly the e-prop synaptic update rule. The second term, which we define as $\widehat{\Gamma_{p q, t}}$, is a synaptically non-local term that is ignored by e-prop. As seen in Eq. 3, our truncation requires maintaining a $\{p, q\}$-dependent double tensor (for $e_{p q, t}$ ) instead of a triple one, thereby reducing the memory cost of RTRL from $O\left(N^{3}\right)$ to $O\left(N^{2}\right)$.

Importantly, we observe that, for the update to synapse $w_{p q}$ in Eq. 3. the terms that depend on cells $j$ only appear under a sum. Therefore, the mechanism updating the synapse $(p q)$ does not need to know the individual terms indexed by $j$. Rather, only their sum suffices. This observation is key in hypothesizing a role for diffuse neuromodulatory signalling as an additional factor in synaptic plasticity. As discussed above, multiple different neuromodulators diffuse in the shared intercellular space [7, 8]; this provides a natural biological substrate for the transmission of weighted, summed signals.

While it is tempting to consider the first factors in $\widehat{\Gamma_{p q, t}}, a_{j, t} w_{j p}$, as the modulatory signal emitted by neuron $j$, the involvement of the synapse from neuron $p$ via $w_{j p}$ and a lack of known mechanisms in calculating this neuron-specific composite signal suggest that this is unlikely to be a biological solution. Instead, inspired by the cell-type-specific (rather than neuron-specific) affinities for peptidergic neuromodulation [7, 8], we propose to approximate the signaling gain $w_{j p}$ in Eq. 3 by the average value $w_{\alpha \beta}$ across its pre- and postsynaptic cell types. More specifically, when postsynaptic cell $j$ belongs to type $\alpha$ and presynaptic cell $p$ belongs to type $\beta$, we approximate neuron-specific weight $w_{j p}$ with cell-type-specific gain $w_{\alpha \beta}=<w_{j p}>_{j \in \alpha, p \in \beta}$. We hypothesize that $w_{\alpha \beta}$ represents the affinity of the G-protein coupled receptors expressed by cells of type $\beta$ to the peptides secreted by cells of type $\alpha$ (Figure 2 aiv, b). Finally, the local diffusion hypothesis discussed in [39] suggests a further approximation, in which this type of signaling is registered only by local synaptic partners and therefore preserves the connectivity 
structure of $w_{j p}$ :

$$
a_{j, t} w_{j p} \approx \begin{cases}a_{j, t} w_{\alpha \beta}, & p \rightarrow j \\ 0, & \text { otherwise }\end{cases}
$$

where $p \rightarrow j$ denotes that there is a synaptic connection from neuron $\mathrm{p}$ to $\mathrm{j}$. In other words, while $a_{j, t}$ is emitted by neuron $j$, its effect on neuron $p$ through diffusion, as part of a sum, is given by Equation 4.

Bringing these together, the gradient estimate at time $t$ due to our learning rule is given as

$$
\begin{aligned}
\left.\frac{\mathrm{d} E}{\mathrm{~d} w_{p q}}\right|_{t} & \left.\approx \frac{\mathrm{d} E}{\mathrm{~d} w_{p q}}\right|_{t, \mathrm{e}-\mathrm{prop}}+\Gamma_{p q, t}, \\
\Gamma_{p q, t} & =\left(\sum_{\alpha \in C} w_{\alpha \beta} \sum_{j \in \alpha, p \rightarrow j} a_{j, t}\right) e_{p q, t-1},
\end{aligned}
$$

where neuron $p$ is of type $\beta, C$ denotes the set of neuronal cell types, $\Gamma_{p q, t}$ approximates the second term in Eq. 3 with cell-type-specific weight averages. Thus, our update rule suggests a new additive term to compute the plasticity update at synapse $p q$ at time $t, \Gamma_{p q, t}$, which calculates multiplicative contributions of the modulatory signal $a_{j, t}$ secreted by neuron $j$, the affinity of receptors of cell type $\beta$ to ligands of type $\alpha, w_{\alpha \beta}$, and the eligibility trace at the synapse $p q, e_{p q, t}$. While Eq. 5 suggests an "online" implementation with the update at $t$, the factor $a_{j, t}$ cannot be calculated causally unless the output is not leaky. Supplementary Note 1 derives an online update for the more general case, which has the same form as Eq. 5 .

In summary, we have proposed a new rule for updating a synapse $w_{p q}$, which we refer to as the multidigraph learning rule, or MDGL, and represent by $\left.\Delta w_{p q}\right|_{M D G L}$. As illustrated in Fig. 2 , this begins with the same basic structure as e-prop, which has the following form:

$$
\left.\Delta w_{p q}\right|_{\text {e-prop }} \propto(\text { Top-down learning signal } \mathrm{p}) \times(\text { eligibility trace } \mathrm{pq}) .
$$

$\left.\Delta w_{p q}\right|_{\text {MDGL }}$ then adds a term $\Gamma_{p q}$, whose form matches that of diffusive communication via a cell-type specific modulatory network. Taking account of the fact that the activity-dependent modulatory signal emission $a_{j, t}$ is a combination of the top-down learning signal received by neuron $j$ and its activity (see Eq. M12 and detailed component breakdown in Supplementary Note 2):

$$
\begin{aligned}
\left.\Delta w_{p q}\right|_{\mathrm{MDGL}} & \left.\propto \Delta w_{p q}\right|_{\mathrm{e}-\text { prop }}+\Gamma_{p q}, \\
\Gamma_{p q} & \approx(\sum_{\alpha \in C}(\text { cell-type-specific gain }) \times \sum_{j \in \alpha} \underbrace{(\text { Mod. signal secreted by } \mathrm{j})}_{(\text {Top-down signal } \mathrm{j}) \times(\text { activity } \mathrm{j})}) \times(\text { eligibility trace pq }) .
\end{aligned}
$$

Thus, the Hebbian eligibility trace is not only compounded with top-down learning signals - as in modern biologically plausible learning rules 25] - but also integrated with cell-type-specific, diffuse modulatory signals. This creates a unified framework that integrates the eligibility trace, local and top-down modulatory signals into a new multi-factor learning rule.

\section{Simulation of multidigraph learning in RSNNs}

To test the efficiency of the MDGL formulation for synaptic plasticity, we apply it to three well-known supervised learning tasks involving temporal processing: pattern generation, a delayed match to sample task, and evidence accumulation. We use two main cell classes, inhibitory (I) and excitatory (E) cells, and obey experimentally observed constraints: cells have synapses that are sign constrained with $80 \%$ of the population being excitatory and the rest inhibitory. We further endow a fraction of the E cells with threshold adaptation [48] to mimic the hierarchical structure of cell types that has been established empirically $[9]$ through its simple example of two main cell types (E and I), one of which has two subtypes (E cells with and without threshold adaptation). Also, refractoriness and synaptic delay are incorporated into all cells' dynamics. Moreover, overall connection probability in the RSNN is also constrained, reflecting the sparse connectivity in neuronal circuits 60, 61. In the main text, all simulated tasks are constrained at 10\% sparsity, and the sparsity parameter is varied in Figure $\mathbf{S} 2$ in the supplementary materials. This connection sparsity is maintained by fixing inactive synapses with 0 weights. Unlike the stochastic rewiring 
bioRxiv preprint doi: https://doi.org/10.1101/2020.1122.393504; this version posted March 6, 2021. The copyright holder for this preprint (which was not certified by peer review) is the author/funder, who has granted bioRxiv a license to display the preprint in perpetuity. It is made available under aCC-BY-NC 4.0 International license.

(Deep R) algorithm [62, 63, our implementation does not allow for rapid and random formation of new synapses after each experience, further increasing biological plausibility.

To study the impact of our learning rule on network performance and dissect the effects of its different components, we train RSNNs using five different approaches for each task (Figure 2): (i) BPTT, which updates weights using exact gradients; (ii) E-prop [48], the state-of-the-art method for biologically plausible training of RSNNs; (iii) TRTRL, the truncated RTRL given in Eq. 3 without the cell-type approximation; (iv) MDGL, which incorporates the cell type approximation given in Eq. 5 using only two cell types; (v) NL-MDGL, a nonlocal version of MDGL, where the gain is replaced by $w_{\alpha \beta}=<w_{j p}>_{j \in \alpha, p \in \beta}$ even for $w_{j p}=0$ so that the modulatory signal diffuses to all cells in the network. While the naive implementation of $a_{j, t}$ depends on future errors in Figures 44, we derive an online approximation in Supplementary Note 1 and demonstrate that it does not lead to performance degradation (Figure S3).

For each learning rule, we train the following network parameters: input, recurrent and output weights. All approaches update the output weights using backpropagation since the nonlocality problem in Eq. 1 applies only to the update of input and recurrent weights. (For updating the weights of a single output layer, random feedback alignment 64 has also been shown to be an effective and biologically plausible solution.)

\section{Multidigraph learning in RSNNs improves efficiency in a pattern generation task}
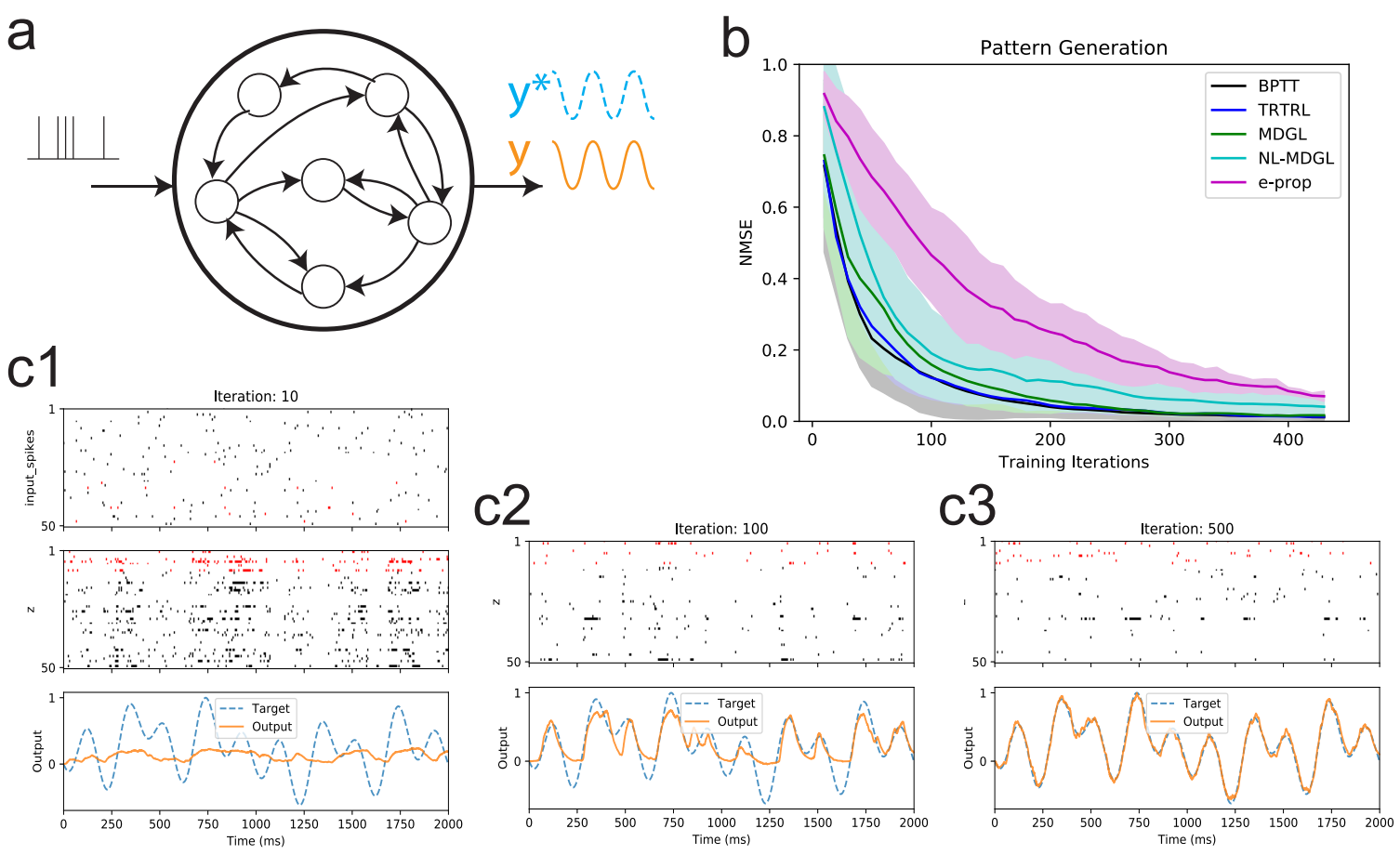

Figure 4: Pattern generation task a) Task setup 65: a network is trained to produce a target output pattern over time. The target is formed from a sum of five sinusoids. b) Normalized mean squared error (NMSE) over training iterations is illustrated for the five learning rules (Fig. 2). Solid lines show the mean and shaded regions show the standard deviation across five runs, with different target output, frozen Poisson input and weight initialization between runs (but fixed within each run). Comparing the performance of e-prop with the MDGL method suggests that the addition of cell-type-specific modulatory signals expedites the learning curve. c) Dynamics of the input, output and recurrent units are shown after 1, 100 and 500 iterations of training using the MDGL method. Raster plots are shown for 50 selected sample cells, and E cells and I cells are color coded using black and red, respectively. All recurrent units have fixed thresholds for this task. Recurrent unit spikes are irregular throughout training. Network output approaches the target as training progresses.

We first trained a RSNN to produce a one-dimensional target output, generated from the sum of five sinusoids, given a fixed Poisson input realization. The task is inspired by that used in 65. We compare the training for the five learning rules illustrated in Fig. 2 and described above.

While the target output and the random Poisson input realization is fixed across training iterations, we change these along with the initial weight for different training runs, and illustrate the learning curve mean and standard 
deviation in Figure $4 \mathrm{~b}$ across five such runs. Here, the learning curve displays the normalized mean squared error (NMSE) between the actual and desired output over training iterations. We observe that our methods, TRTRL (blue) and MDGL (green), reduce NMSE faster over training iterations compared to e-prop (magenta). Also, removing the locality of the modulatory signal (NL-MDGL) degraded the efficiency, although learning with spatially non-specific modulation still outperformed that without the modulatory signal (e-prop).

We highlight the fact that approximating the nonlocal learning rule of TRTRL with diffuse, modulatory signaling among two cell types results in only a moderate degradation of performance. To better understand this observation, we conduct an analysis of the similarity between the $\widehat{\Gamma_{p q}}$ term computed by TRTRL in Eq. 3 and its cell-type-based approximation $\Gamma_{p q}$ computed by MDGL. We quantify this via the alignment angle, which describes the similarity between the directions of the two vectors (Supplementary Note 3. Supplementary Table S1). The significant alignment between these two signals, despite the underlying vectors lying in very high dimensional spaces of synaptic weights, demonstrates that the two methods compute similar gradients, thus shedding light on the similarity between their learning curves.

Figure 4 illustrates the input, recurrent network activity, and target versus actual outputs trained using the MDGL method after training for 10,100 and 500 iterations. Recurrent unit spikes appear to be reasonably irregular over time, broadly consistent with what is typically observed biologically [66, 67], with no obvious patterns of system-wise synchrony throughout training. Finally, the network output approaches the target as training progresses (lower panels).

\section{Multidigraph learning in RSNNs improves efficiency in a delayed match-to-sample task}

To elucidate how neural networks with the learning rules at hand can be trained to integrate a history of past cues to generate responses that impact a reward delivered later, we considered a special case of the delayed match to sample task described in 68. Here, two cue alternatives are encoded by the presence and absence of input spikes. Our implementation of the task began with a brief fixation period (no cues) followed by two sequential cues, each lasting $0.15 \mathrm{~s}$ and separated by a $0.75 \mathrm{~s}$ delay (Figure $5 \mathrm{z}$ ). A cue of value 1 was represented by $40 \mathrm{~Hz}$ Poisson spiking input, whereas a cue of value 0 was represented by the absence of input spiking. The network was trained to output 1 (resp. 0 ) when the two cues have matching (resp. non-matching) values. That is, the RSNN was trained to remember the first cue and learn to compare it with the second cue delivered at a later time.

Figure 5 displays the learning curve for novel (test) inputs for the five plasticity rules described above. We observe that the same general conclusions as for the pattern generation task hold here: TRTRL (blue) and MDGL (green) outperform e-prop (magenta). The performance degrades when we remove the neighborhood specificity of the modulatory signal (NL-MDGL, cyan). Moreover, alignment angles between MDGL, TRTRL, and e-prop are also similar to those of the pattern generation task (Supplementary Tables S1 and S2). We illustrate the network dynamics after training using the MDGL method for 100, 500 and 2000 iterations in Figure 5 $51-\mathrm{c} 3$. We observe that as training progresses, the network output decides on the correct prediction with greater confidence, i.e. the output neuron corresponding to the correct target approaches a value of 1 . Figure S5 shows that these observations also hold for nonzero firing representation of the second cue alternative.

\section{Multidigraph learning in RSNNs improves efficiency in an evidence accumulation task}

Finally, we study an evidence accumulation task [70, 69, which involves integration of several cues in order to produce the desired output at a later time: an agent moves along a straight path while encountering a series of sensory cues presented either on the right or left side of a track (Figure 6a). Each cue is represented by $40 \mathrm{~Hz}$ Poisson spiking input for $100 \mathrm{~ms}$ and cues are separated by $50 \mathrm{~ms}$. After a delay of $850 \mathrm{~ms}$ when the agent reaches a T-junction, it has to decide if more cues were received on the left or right. Thus, this task requires not only recalling past cues, but also being able to count the cues separately for each side and then process these cues for a reward delivered at a much later time. While e-prop can be used to train a RSNN to solve this task [48], it requires (i) significantly more training iterations than BPTT, and (ii) rapid and stochastic creation/pruning of synapses after each iteration 62. Therefore, we test our learning rule in Figure 6 to see if the addition of diffuse modulatory signals can indeed bring the learning curve closer to BPTT, without relying on stochastic rewiring.

Figure 6b and Supplementary Tables S1 and S2 demonstrate that all of our conclusions in the previous two experiments continue to hold in this task: MDGL is closest to BPTT, and NL-MDGL gives relatively degraded performance yet still outperforms e-prop. Figure S10 further shows that gradients approximated by TRTRL and MDGL are more similar to the exact gradients compared to e-prop for the previous two tasks as well as this one. We also observe that the timing of recurrent unit spiking patterns tightly follows that of input cue presentation, 
bioRxiv preprint doi: https://doi.org/10.1101/2020.11.22.393504; this version posted March 6, 2021. The copyright holder for this preprint (which was not certified by peer review) is the author/funder, who has granted bioRxiv a license to display the preprint in perpetuity. It is made available under aCC-BY-NC 4.0 International license.

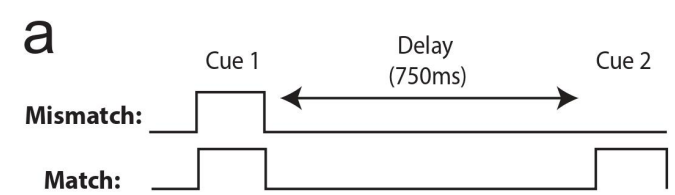

b

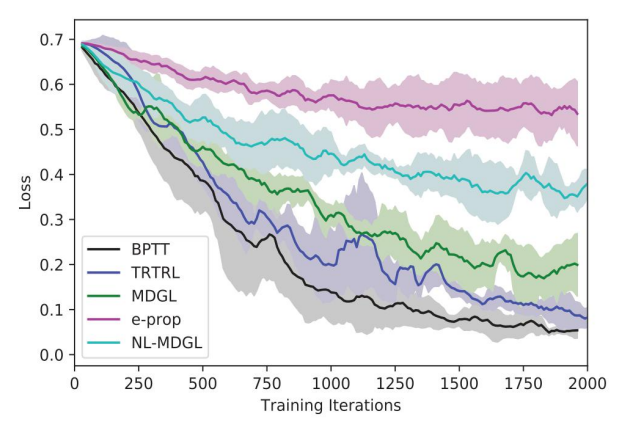

c1
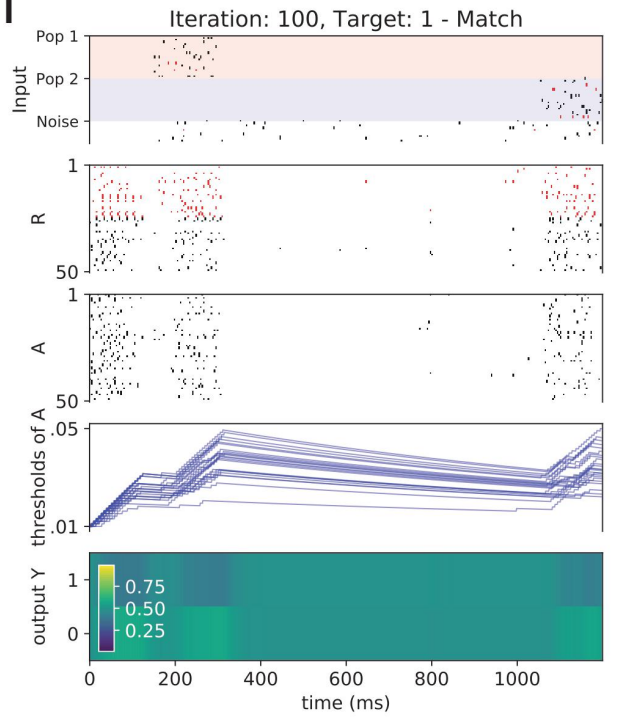

c2
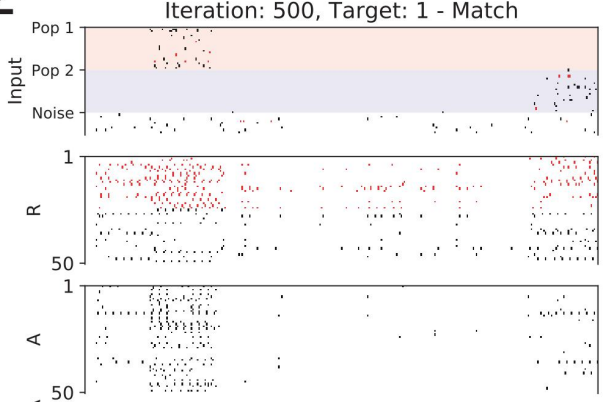

$\varangle 50$
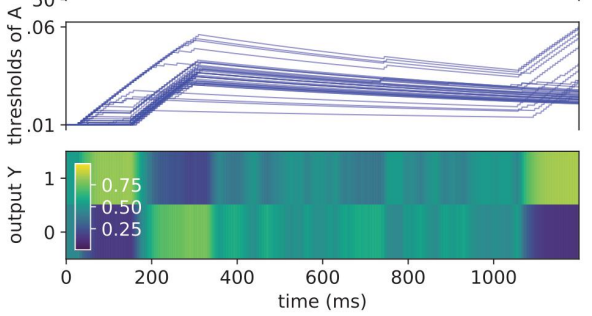

c3
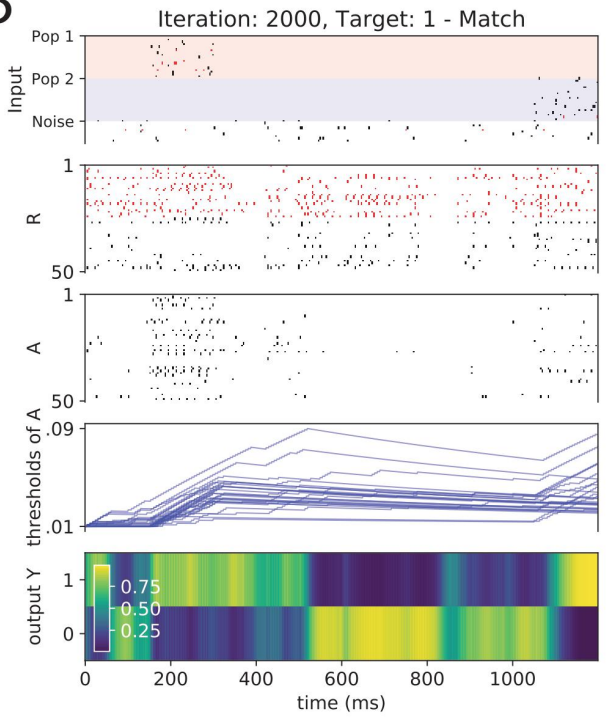

Figure 5: Application of the cell-type-specific modulatory signals to the delayed match-to-sample task. a) Setup of a special case of the delayed match to sample task, where two cue alternatives are represented by the presence and absence of input spikes. b) Learning curves of aforementioned training methods in Figure $2 \mathrm{a}$. Loss is obtained from test data using different realizations of random Poisson input than in training data. Solid lines show the mean and shaded regions show the standard deviation across five runs, with a different weight initialization for each run. Comparing the performance of e-prop with the MDGL method suggests that the addition of cell-type-specific modulatory signals improves learning. c) Network dynamics of an example trial after 100,500 and 2000 iterations of training using the MDGL method are illustrated in $\mathrm{c} 1, \mathrm{c} 2$ and $\mathrm{c} 3$, respectively. To emphasize the change in dynamics over training iterations, we used the same cue pattern for the illustrations. Again, E cells and I cells are color coded using black and red, respectively. For this task, both recurrent units with adaptive threshold (labeled as A) and without (labeled as R) are involved [48]. Threshold dynamics of sample neurons are illustrated. The network makes the correct prediction with greater confidence as training progresses. Figure $\mathrm{S} 5$ shows similar results with the nonzero firing representation of the second cue alternative as well.

suggesting that the network is taking immediate action ("counting") for each cue. We illustrate the network dynamics after training using the MDGL method for 100, 500 and 2000 iterations in Figure 661-c3. We observe that as training progresses, the network output decides on the correct prediction with greater confidence.

While the adaptive firing threshold already provides a form of memory, Figure S6a shows that both threshold adaptation and recurrence are needed for this memory task. (i.e., adaptive threshold alone is not sufficient.) Moreover, Figure S6 $\mathrm{p}$ shows that this conclusion is not sensitive to the precise fraction of the adaptive population. 
bioRxiv preprint doi: https://doi.org/10.1101/2020.11.22.393504; this version posted March 6, 2021. The copyright holder for this preprint (which was not certified by peer review) is the author/funder, who has granted bioRxiv a license to display the preprint in perpetuity. It is made available under aCC-BY-NC 4.0 International license.

a

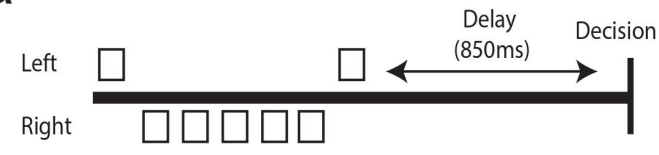

$\mathrm{b}$

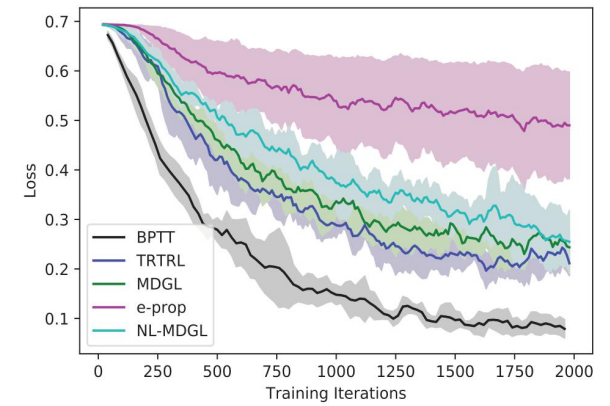

c1
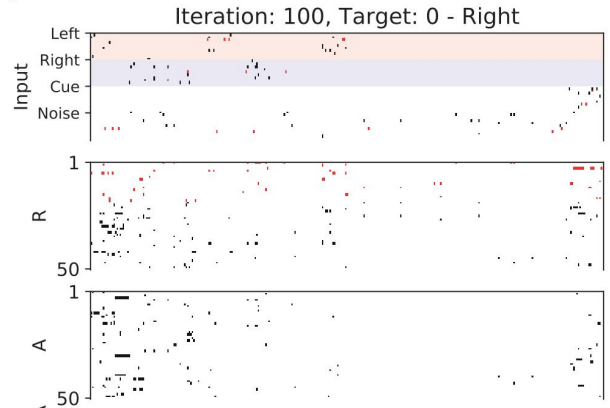

$\varangle 50$
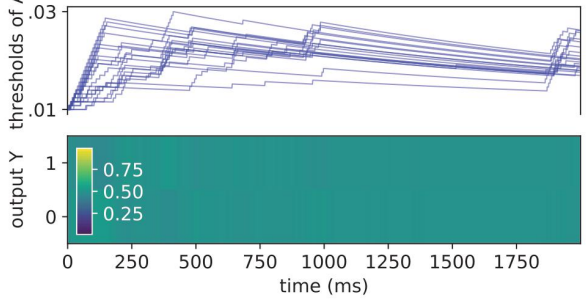

c2
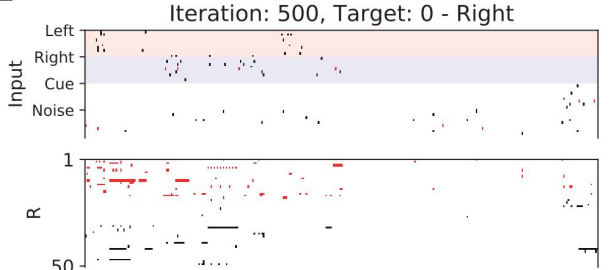$$
50
$$
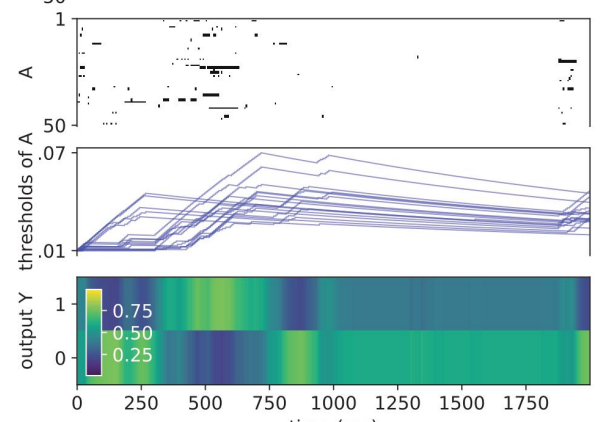

C3
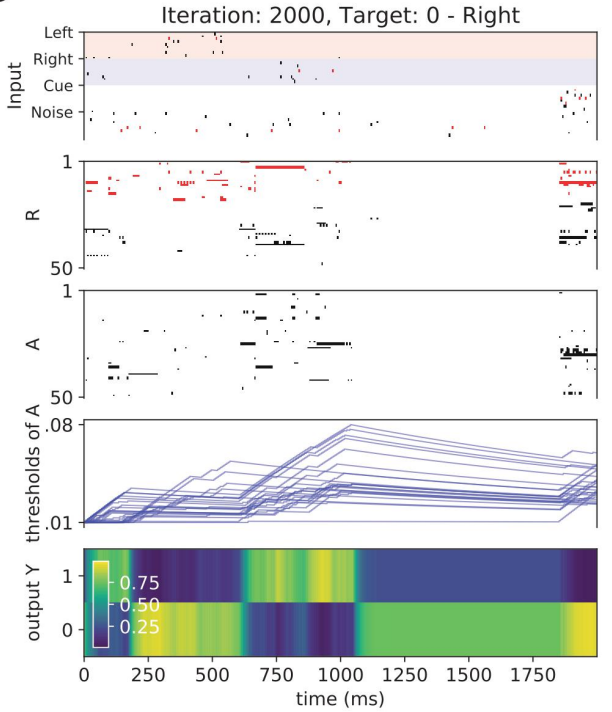

Figure 6: Application to the evidence accumulation task. a) Setup of the task inspired by 69. 70 . b) Learning curves of aforementioned training methods in Figure 2 a. Loss is obtained from test data using different realizations of random Poisson input than in training data. Solid lines show the mean and shaded regions show the standard deviation across five runs, with a different weight initialization for each run. Comparing the performance of e-prop with the MDGL method suggests that the addition of cell-type-specific modulatory signals improves learning. c) Network dynamics (Input spikes, recurrent unit spikes and readout) of an example trial after 100, 500 and 2000 iterations of training using the MDGL method are illustrated in c1, c2 and c3, respectively. To emphasize the change in dynamics over training iterations, we used the same target direction for the illustrations. Similar to the previous task, both recurrent units with adaptive threshold (labeled as A) and without (labeled as R) are involved [48, and threshold dynamics of sample neurons are illustrated. The network makes the correct prediction with greater confidence as training progresses. For all methods, results were obtained without using stochastic rewiring, which allows for random formation of new synapses in each experience (Deep R) 62 63 .

\section{Multidigraph learning in RSNNs produce fast synaptic signaling and slow modulatory signaling}

Owing to their diffusive nature and the fact that GPCRs act on significantly longer time scales than receptors for many synaptic neurotransmitters $[8]$, modulatory signaling channels are limited to be slower and smoother (i.e., have lower bandwidth) than direct synaptic channels. Since our model does not explicitly limit the communication bandwidths of either of these channels, comparing the frequency content of these two channels offers an important check for biological plausibility. It also provides a test of our assumption that the summation over $j$ in Eq. 3 acts as a smoothing operation, enabling subsequent approximation with diffuse modulatory signaling. Figure 7 demonstrates 
that the modulatory input indeed has significantly lower frequency content than the synaptic input, for all three tasks studied above.

In sum, our results on the slow timescales of cell-type averaged signaling - together with the gradient alignment results mentioned above (presented in supplementary materials) - help to illustrate why modulatory signaling that is non-specific across both space and time can nonetheless lend significant improvement to network learning curves. This form of signaling removes the need for specifically tuned, reciprocal physical contacts and signaling among cell pairs and hence biologically implausible features of anatomical organization that plague solutions to the credit assignment problem via pairwise synaptic communication alone.
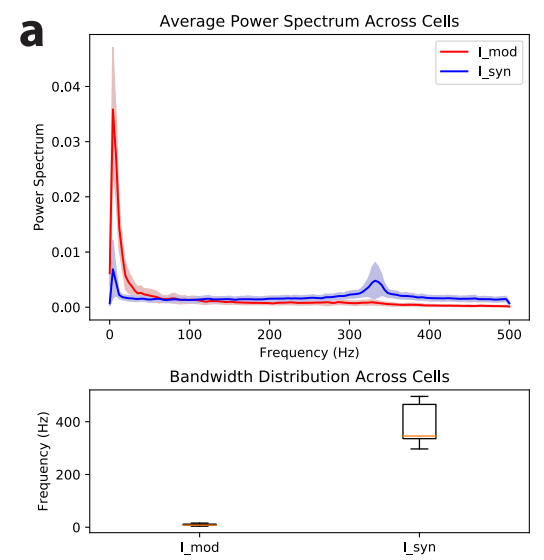
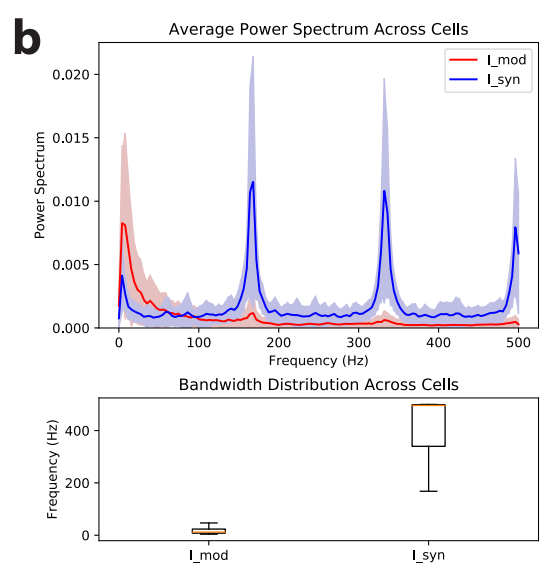
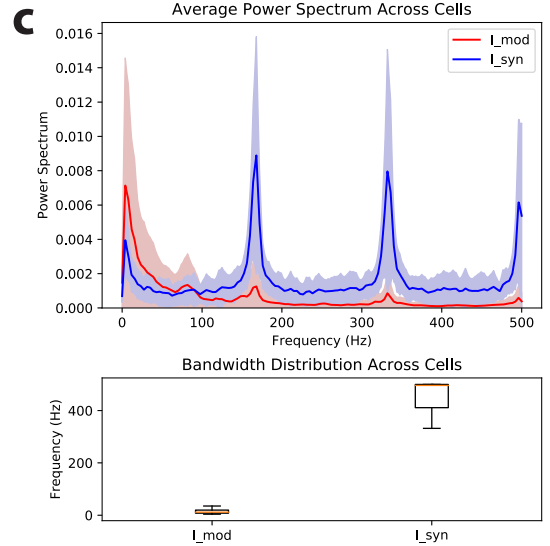

Figure 7: Frequency distribution of modulatory versus synaptic inputs. Comparing the power spectrum and bandwidth distributions between modulatory and synaptic inputs for a) pattern generation, b) delayed match to sample and c) evidence accumulation tasks. In the top panel, the solid lines denote the average and shaded regions show the standard deviation of power spectrum across recurrent cells. In the bottom panel, box plots show the minimum, lower quartile, median, upper quartile and maximum bandwidth across the cells. Here, the bandwidth is quantified by the $3 \mathrm{~dB}$ frequency, where the power halves compared to the peak. Nyquist's theorem dictates that the simulation interval of $1 \mathrm{~ms}$ limits the maximum frequency to $500 \mathrm{~Hz}$. Modulatory input is the total cell-typespecific modulatory signals detected by each cell $p$, defined as $I_{m o d, p}:=\sum_{\alpha \in C} w_{\alpha \beta} \sum_{j \in \alpha, p \rightarrow j} a_{j, t}$ (Eq. 55. Synaptic input is the total input received through synaptic connections by each cell $j$, defined as $I_{s y n, j}:=\sum_{l \neq j} w_{j l} z_{l, t}+\sum_{p} w_{j m}^{\mathrm{IN}} x_{m, t+1}$ (Eq. M1). The naive implementation of the modulatory signal, $a_{j, t}$, depends on future errors unless the output is not leaky (Eq. M12 in Methods, Supplementary Note 1). Thus, we repeated the spectral analysis for the online implementation of modulatory signaling in Eq. S3 and observed similar conclusions (Figure S4. Here, the plots are obtained at the end of training (after 500 iterations for pattern generation and 2000 iterations for delayed match to sample and evidence accumulation tasks), but similar trends are observed at other training snapshots.

\section{Discussion}

In this paper, we presented a normative theory of temporal credit assignment and an associated biologically plausible learning rule where neurons are allowed to communicate via not only the synaptic connections but also a secondary, non-specific "modulatory" channel. In particular, we explained how the recent observation of wide-spread and cell-type-specific modulatory signaling [8], when integrated with synaptic networks to interconnect cortical neurons, can promote efficient learning. We demonstrated that the associated biologically plausible learning rule achieves performance close to that of ideal, but biologically unrealistic, rules on multiple in silico learning tasks - pattern generation, delayed match-to-sample, and evidence accumulation - that require temporal credit assignment over timescales spanning seconds. These experiments used sparsely and recurrently connected neural networks of signconstrained spiking neurons, capturing some of the well-studied aspects of brain architecture and computation 60 , 61. All simulation results were obtained without using rapid stochastic rewiring, which allows for random formation of new synapses in each experience 62,63 . Thus, learning with local modulatory signals can relax the need for rapid and random creation/pruning of synapses. While our demonstrations use the supervised learning paradigm, the same machinery can be easily applied to reinforcement learning tasks [48].

The existence of multiple directed connections between neurons suggests a multidigraph view of brain connectivity, in which each neuron is connected by multiple different connection types (Figures 1 and 2b) 8]. Therefore, we call our learning paradigm, multidigraph learning. MDGL begins with the same basic structure as the RFLO [47] and e-prop 48 learning rules, but adds the cell-type-specific local modulatory network to achieve a better approximation 
to ideal synaptic weight updates. While we did not constrain the temporal dynamics of this secondary signaling mechanism, we found that the cell-type-specific broadcast channel communicated at significantly lower frequencies in all our experiments, in agreement with well-known properties of diffusive modulatory signaling.

Our model posits that top-down learning signals can influence the secretion of cell-type-based modulatory signals (Eq. 7 and Supplementary Note 2). As one possible mechanism for this, dopamine can alter neuronal firing [71], which can then impact neuropeptide release in an activity-dependent manner [39]. This finding predicts that cell-type-specific signaling carries information about top-down learning signals, so their levels can reflect the learning progress. Indeed, our computational experiments suggest that the level of modulatory input decreases over training iterations and sharply rises in response to changes in task condition (Figure S7). Rapidly maturing technologies on in-vivo monitoring of neuromodulators 72, 73, 41, suggest that such predictions of our model can be tested experimentally.

The nature of "intermediate" cells [9], whose phenotypes appear to be a mixture of "pure" cell types is a key problem in cell types research. Our findings explain the existence of such phenotypes from a connectivity perspective: while average connectivities between types $w_{\alpha \beta}$ remain relatively constant during training, connectivities of individual cells can deviate significantly from those averages (Figure S8). We also hypothesized a link between abstract cell type-based connectivities and modulatory receptor efficacies. From this perspective, how tightly the individual synaptic weights and cell-type-specific (but not neuron specific) receptor efficacies should be coupled (i.e., co-adaptation) may be explored in future work. Figure S9 suggests that the effect of imprecise GPCR efficacies $\left(w_{\alpha \beta}\right)$ on the performance is task-dependent, and a wide range of fixed $w_{\alpha \beta}$ values can enable effective learning in some, but not all, tasks.

As a proof of concept, we tested a minimal implementation of MDGL: there are just two local modulatory types mapping to the two main cortical cell classes (E and I), and the fine-grained E cell subtypes (those with and without firing threshold adaptation) were grouped into one modulatory type. Even the minimal implementation led to a marked improvement in learning efficiency compare to existing biological learning rules. However, brain cells are extremely diverse $74,75,76,77,78,79$ with a matching diversity in the expression of peptidergic genes; at least 18 NP precursor and 29 NP-GPCR genes were reported to have widespread and cell-type-specific expression in the mouse cortex [7]. Therefore, further studies can investigate how MDGL scales to harder tasks and examine the interplay of task complexity and cell diversity. A related point is that, here, we did not study the potential diversity of time scales across different types of NP signals 39 . Future work may investigate the role of distinct timescales in local modulatory signaling.

Our MDGL learning rule assumes that cell-type-based modulatory signals diffuse locally such that they are registered only by local synaptic partners of the source neuron. On the other hand, NL-MDGL assumes that these signals diffuse to all cells of a given type in the network. The biological reality may lie somewhere in between. Studying the extent of the local diffusion hypothesis, where neuropeptide signals act on both synaptic partners and nonsynaptic partners in the vicinity 39 may suggest new learning rules in the future and deepen our understanding of their underlying mechanisms.

Learning rules often explicitly optimize a loss function, and the direction of the steepest descent in the loss function can be identified from the exact gradient, when available. Rules that follow this exact gradient, RTRL and BPTT, are well established, but are not biologically plausible and have unmanageably vast memory storage demands. However, a growing body of studies have demonstrated that learning rules that only partially follow the gradient, while alleviating some of these problems of the exact rules, can still lead to desirable outcomes [80, 81]. An example is the seminal concept of feedback alignment [64], which rivals backpropagation on a variety of tasks even using random feedback weights for credit assignment. In addition, approximations to RTRL have been proposed 82,83 , 84, 85] for efficient online learning in RNNs. Reference [47] also derived a $O\left(N^{2}\right)$ rule that maintained only a rank-2 tensor for training rate-based RNNs by fully truncating the inter-cellular dependencies involving cells other than preand postsynaptic neurons. Instead of full truncation, SnAp- $n 54,55$ stores the Jacobian $\frac{\mathrm{d} s_{j, t}}{\mathrm{~d} w_{p q}}$ for all $j$ influenced by $w_{p q}$ within $n$ time steps. SnAp-1 is effectively the truncation in [47] and the performance of SnAp- $n$ increases with $n$, resonating with our improved performance when more terms of the exact gradient are included. However, SnAp- $n$ needs to maintain a triple tensor for $n \geq 2$. While our approximation is similar to SnAp-2, it only needs to store the double tensor $\frac{\mathrm{d} s_{p, t}}{\mathrm{~d} w_{p q}}$ since we further constrain inter-cellular signaling via diffuse pathways organized by cell types. Thus, our model further advances the efficacy of approximated gradient-based learning methods and continues the line of research in energy-efficient on-chip learning through spike-based communications [86, 87]. Such efficient approximations of the gradient computation can be especially important as artificial networks become ever larger and are used to tackle ever more complex tasks under both time and energy efficiency constraints.

Our work fits under the wide umbrella of neoHebbian multi-factor learning 21, 26. This theory posits additional 
modulatory factors, which combine with factors based on pre- and postsynaptic activity to determine synaptic plasticity. Molecules that could keep a memory trace of classical Hebbian activity include calcium ions and activated CaMKII enzymes [88] although the biological underpinning remains enigmatic. The additional modulatory factors could arise from a diverse set of signals found in the brain. Most existing theoretical models conceive these factors as top-down learning signals coming from distant brain regions, such as dopamine, noradrenaline and neural firing [89]. Here, we took top-down learning signals to be cell-specific rather than global, which is justified in part by recent reports that dopamine signals $[69]$ and error-related neural firing 89 can be specific to a population of neurons [48]. On the other hand, this specificity could be removed by random feedback alignment 64 or via an approach similar to our cell-type-specific weight approximation in Eq. 4 (Supplementary Note 2). In addition to top-down learning signals, we add further modulatory factors, released from within the recurrent network performing the computation at hand. These signals represent locally secreted modulatory signals, such as those carried by neuropeptides, released from nearby cells of a given type. Such signalling is abundantly present in the brain but had yet to be interpreted in terms of the credit assignment problem. Similar to dopaminergic regulation of plasticity [71, neuropeptides have also been shown to modulate plateau potential, which could alter the amount of plasticity [90].

Our work suggests that multiple cell-type-specific, diffuse and relatively slow modulatory signals should be considered as possible bases for credit assignment computations. Though inspiration for the present work came primarily from new transcriptomic data on local NP signaling in neocortex [7, 8, it is quite possible that other cell-type-specific neuromodulators could likewise contribute to credit assignment. Many of these alternative agents act, as do NPs, via GPCRs (e.g., the monoamines, amino acids, acetylcholine and endocannabinoids, as noted above), but our multidigraph template might even apply to other neuronally secreted neuromodulators, such as the neurotrophins and cytokines, that act via different classes of receptor [91, 92. While experimental tests of such hypotheses have not seemed feasible up until now, emerging methods for genetically addressed measurement of various neuromodulatory signals in specific cell types $[7,93,94]$ are now bringing the necessary critical tests within reach (e.g. [41]).

\section{Methods}

Neuron Model: We consider a discrete-time implementation of RSNNs. The network, as shown in Figure 3 a, denotes the observable states, i.e. spikes, as $z_{t}$ at time $t$, and the corresponding hidden states as $s_{t}$. For LIF cells, the state $s_{t}$ corresponds to membrane potential and the dynamics of those states are governed by

$$
\begin{aligned}
z_{j, t} & =H\left(s_{j, t}-v_{\mathrm{th}}\right) \\
s_{j, t+1} & =\eta s_{j, t}+(1-\eta)\left(\sum_{l \neq j} w_{j l} z_{l, t}+\sum_{p} w_{j m}^{\mathrm{IN}} x_{m, t+1}\right)-z_{j, t} v_{\mathrm{th}} \\
y_{k, t} & =\kappa y_{k, t-1}+(1-\kappa) \sum_{j} w_{k j}^{\mathrm{OUT}} z_{j, t}+b_{k}^{\mathrm{OUT}}
\end{aligned}
$$

where $s_{j, t}$ denotes the membrane potential for neuron $j$ at time $t, v_{\text {th }}$ denotes the spiking threshold potential, $\eta=e^{-d t / \tau_{m}}$ denotes the leak factor for simulation time step $d t$ and membrane time constant $\tau_{m}, w_{l j}$ denotes the weight of the synaptic connection from neuron $j$ to $l, w_{j m}^{\mathrm{IN}}$ denotes the efficacy of the connection between the input neuron $m$ and neuron $j$, and $H$ denotes the Heaviside step function. For the output, $w_{k j}^{\text {OUT }}$ denotes the efficacy of the connection from neuron $j$ to output neuron $k$, and $b_{k}^{\text {OUT }}$ denotes the bias of the $k$-th output neuron.

Following references [63, 48, which implemented adaptive threshold LIF (ALIF) units 95] and observed that this neuron model improves computing capabilities of RSNNs relative to networks with LIF neurons, we also include ALIF cells in our model. In addition to the membrane potential, ALIF cells have a second hidden variable, $b_{t}$, governing the adaptive threshold. The spiking dynamics of both LIF and ALIF cells can be characterized by the following set of equations:

$$
\begin{aligned}
s_{j, t+1} & =\eta s_{j, t}+(1-\eta)\left(\sum_{l \neq j} w_{j l} z_{l, t}+\sum_{p} w_{j m}^{\mathrm{IN}} x_{m, t+1}\right)-z_{j, t} v_{\mathrm{th}} \\
z_{j, t} & =H\left(s_{j, t}-A_{j, t}\right) \\
A_{j, t} & =v_{\mathrm{th}}+\beta b_{j, t} \\
b_{j, t} & =\rho b_{j, t-1}+(1-\rho) z_{j, t-1},
\end{aligned}
$$


where the voltage dynamics in Eq. M3 is the same as Eq. M1. A spike is generated when the voltage $s_{j, t}$ exceeds the dynamic threshold $A_{j, t}$. Parameter $\beta$ controls how much adaptation affects the threshold and state $b_{j, t}$ denotes the variable component of the dynamic threshold. The decay factor $\rho$ is given by $e^{-d t / \tau_{b}}$ for simulation time step $d t$ and adaptation time constant $\tau_{b}$, which is typically chosen on the behavioral task time scale. For regular LIF neurons without adaptive threshold, one can simply set $\beta=0$.

Network output and loss function: Dynamics of leaky, graded readout neurons was implemented as $y_{k, t}=\kappa y_{k, t-1}+(1-\kappa) \sum_{j} w_{k j}^{\mathrm{OUT}} z_{j, t}+b_{k}^{\mathrm{OUT}}$. The $(1-\kappa)$ factor in the second term was dropped in writing for readability but kept during the actual implementation. Here, $\kappa \in(0,1)$ defines the leak and $\kappa=e^{-d t / \tau_{O U T}}$ for output membrane time constant $\tau_{O U T}$. We provide the online implementation for this readout convention in Supplementary Note 1 .

We quantify how well the network output matches the desired target using error function $E$. For regression tasks such as pattern generation, we use $E=\sum_{k, t}\left(y_{k, t}^{*}-y_{k, t}\right)^{2}$ given time-dependent target $y_{k, t}^{*}$. For classification tasks such as delayed match to sample and evidence accumulation, $E=-\sum_{k, t} \pi_{k, t}^{*} \log \pi_{k, t}$ with one-hot encoded target $\pi_{k, t}^{*}$ and predicted category probability $\pi_{k, t}=\operatorname{softmax}_{k}\left(y_{1, t}, \ldots, y_{N_{O U T}, t}\right)=\exp \left(y_{k, t}\right) / \sum_{k^{\prime}} \exp \left(y_{k^{\prime}, t}\right)$. We provide all simulation and training parameters in Supplementary Note 3

Notation for Derivatives: There are two types of computational dependencies in RSNNs: direct and indirect dependencies. For example, variable $w_{p q}$ can impact state $s_{p, t}$ directly through Eq. M1 as well as indirectly via its influence through other cells in the network. We distinguish direct dependencies versus all dependencies (including indirect ones) using partial derivatives $(\partial)$ versus total derivatives $(d)$.

Differentiation in RSNNs: As mentioned, we study iterative adjustment of all synaptic weights using gradient descent on loss $E$ :

$$
\begin{aligned}
w_{p q, \text { new }} & =w_{p q, \text { old }}-\lambda \Delta w_{p q}, \\
\Delta w_{p q} & =\frac{\mathrm{d} E}{\mathrm{~d} w_{p q, \text { old }}},
\end{aligned}
$$

where $\lambda$ denotes the learning rate, and the gradient of the error with respect to the synaptic weights must be calculated. As explained before in Results, gradient-based learning rules, BPTT and RTRL, calculate this by unwrapping the RSNN dynamics over time. While the two algorithms yield equivalent results, we focus our analysis on RTRL, since its computational dependency graph of states is causal. Using RTRL, we factor the error gradient as follows:

$$
\begin{aligned}
\frac{\mathrm{d} E}{\mathrm{~d} w_{p q}} & =\sum_{j, t} \frac{\partial E}{\partial z_{j, t}} \frac{\mathrm{d} z_{j, t}}{\mathrm{~d} w_{p q}} \\
\frac{\mathrm{d} z_{j, t}}{\mathrm{~d} w_{p q}} & =\frac{\partial z_{j, t}}{\partial s_{j, t}} \frac{\mathrm{d} s_{j, t}}{\mathrm{~d} w_{p q}}
\end{aligned}
$$

following the derivation notation explained above. The factor $\frac{\partial E}{\partial z_{j, t}}$ in Eq. M8 is related to the top-down learning signal $L_{j, t}:=\sum_{k} w_{k j}^{O U T}\left(y_{k, t}-y_{k, t}^{*}\right)[48$. Supplementary Notes 1 [2] show that the leak term of the output neurons makes these two terms different, and derives an online implementation that uses $L_{j, t}$. For readability, however, we simply refer to $\frac{\partial E}{\partial z_{j, t}}$ as top-down learning signal in the main text, leaving the detailed expansion of derivatives to the supplementary materials.

We now discuss the second factor in Eq. M8 i.e. $\frac{\mathrm{d} z_{j, t}}{\mathrm{~d} w_{p q}}$. This is expanded in two factors in Eq. M9. The first factor, $h_{j, t}:=\frac{\partial z_{j, t}}{\partial s_{j, t}}$ is problematic to compute for spiking neurons due to the discontinuous step function $H$ in Eq. M3 whose derivative is not defined at 0 and is 0 everywhere else. We overcome this issue by approximating the decay of the derivative using a piece-wise linear function $63,86,96,87$. Here, the pseudoderivative $h_{j, t}$ is defined as follows:

$$
\begin{aligned}
h_{j, t} & =\frac{\mathrm{d} z_{j, t}}{\mathrm{~d} s_{j, t}} \\
& \approx \gamma \max \left(0,1-\left|\frac{s_{j, t}-A_{j, t}}{v_{\mathrm{th}}}\right|\right),
\end{aligned}
$$

The dampening factor $\gamma$ (typically set to 0.3 ) dampens the increase of backpropagated errors in order to improve the stability of training very deep (unrolled) RSNNs [63]. Throughout this study, refractoriness is implemented as in [48, where $h_{j, t}$ and $z_{j, t}$ are fixed at 0 after each spike of neuron $j$ for 2 to $5 \mathrm{~ms}$. 
The last factor, $\frac{\mathrm{d} s_{j, t}}{\mathrm{~d} w_{p q}}$, accounts for both spatial and temporal dependencies in RSNNs and can be obtained recursively using Eq. 1. As seen from Eq. 1 and explained in Results, this factor poses key issues to biological plausibility and computational cost.

Cell-type-specific signaling implementation: As introduced in Eq. 5 , activity-dependent modulatory signal emitted by neuron $j$ at time $t$, an important component of MDGL, is defined as

$$
a_{j, t}=\frac{\partial E}{\partial z_{j, t}} \frac{\partial z_{j, t}}{\partial s_{j, t}} .
$$

As defined, $a_{j, t}$, is a package of two components: $\frac{\partial E}{\partial z_{j, t}}$, which is referred to as the top-down signal 48 , and $h_{j, t}=\partial z_{j, t} / \partial s_{j, t}$, which is the pseudo-derivative of spiking activity as a function of cell $j$ 's membrane potential explained above.

Eligibility Trace Implementation: As introduced in Eq. 5. eligibility trace, another important component of MDGL, is defined as

$$
\begin{aligned}
e_{p q, t} & :=\frac{\partial z_{p, t}}{\partial s_{p, t}} \frac{\mathrm{d} s_{p, t}}{\mathrm{~d} w_{p q}}, \\
\frac{\mathrm{d} s_{p, t}}{\mathrm{~d} w_{p q}} & =\frac{\partial s_{p, t}}{\partial w_{p q}}+\frac{\partial s_{p, t}}{\partial s_{p, t-1}} \frac{\mathrm{d} s_{p, t-1}}{\mathrm{~d} w_{p q}},
\end{aligned}
$$

where Eq. M14 follows directly from Eq. 2. $\frac{\mathrm{d} s_{p, t}}{\mathrm{~d} w_{p q}}$ can be obtained recursively and is referred to as the eligibility vector [48]. $e_{p q, t}$ keeps a fading memory of activity pertaining to presynaptic cell $q$ and postsynaptic cell $p$. Here, we briefly explain its implementation by expanding the factors in Eqs. M13 and Eq. M14 for both LIF and ALIF cells.

For LIF cells, there is no adaptive threshold so the hidden state consists only of the membrane potential. Thus, we have factors $\frac{\partial z_{p, t}}{\partial s_{p, t}}=h_{j, t}$ with pseudo-derivative $h_{j, t}$ defined in Eq. M10, $\frac{\partial s_{p, t}}{\partial w_{p q}}=z_{q, t-1}$ and $\frac{\partial s_{p, t+1}}{\partial s_{p, t}}=\eta-v_{\mathrm{th}} h_{j, t}$ following Eq. M1.

For ALIF cells, there are two hidden variables so the eligibility vector is now a two dimensional vector $\frac{\mathrm{d} s_{p, t}}{\mathrm{~d} w_{p q}}=$ $\left[\frac{\mathrm{d} s_{p, t}^{v}}{\mathrm{~d} w_{p q}}, \frac{\mathrm{d} s_{p, t}^{b}}{\mathrm{~d} w_{p q}}\right] \in \mathbb{R}^{2 x 1}$ pertaining to membrane potential $v_{p, t}$ and adaptive threshold state $b_{p, t}$. Following Eq. M3 one can obtain factors $\frac{\partial z_{p, t}}{\partial s_{p, t}}=\left[\frac{\partial z_{p, t}}{\partial v_{p, t}}, \frac{\partial z_{p, t}}{\partial b_{p, t}}\right]=\left[h_{j, t},-\beta h_{j, t}\right] \in \mathbb{R}^{1 x 2}, \frac{\partial s_{p, t}}{\partial w_{p q}}=\left[z_{q, t-1}, 0\right] \in \mathbb{R}^{2 x 1}$ and $\frac{\partial s_{p, t}}{\partial s_{p, t-1}}$ is now a 2 -by-2 matrix:

$$
\frac{\partial s_{p, t}}{\partial s_{p, t-1}}=\left[\begin{array}{cc}
\frac{\partial v_{p, t}}{\partial v_{p, t-1}} & \frac{\partial v_{p, t}}{\partial b_{p, t-1}} \\
\frac{\partial b_{p, t}}{\partial v_{p, t-1}} & \frac{\partial b_{p, t}}{\partial b_{p, t-1}}
\end{array}\right]=\left[\begin{array}{cc}
\eta-v_{\text {th }} h_{j, t} & v_{\text {th }} \beta h_{j, t} \\
(1-\rho) h_{j, t} & \rho-(1-\rho) \beta h_{j, t}
\end{array}\right] \in \mathbb{R}^{2 x 2} .
$$

Thus, the eligibility trace $e_{p q, t}$ would be scalar valued regardless of the dimension of the eligibility vector.

Firing Rate Regularization: In addition to accuracy optimization described above, we added a firing rate regularization term $E_{\text {reg }}$ to the loss function to ensure sparse firing [48]:

$$
E_{\mathrm{reg}}=\frac{1}{2} c_{\mathrm{reg}} \sum_{j}\left(f_{j}^{\mathrm{av}}-f_{j}^{\mathrm{target}}\right)^{2}
$$

where $f_{j}^{\text {target }}$ and $f_{j}^{\text {av }}=\frac{1}{T} \sum_{t} z_{j, t}$ are the desired and actual average firing rate for cell $j$, respectively, and $c_{\text {reg }}$ is a positive coefficient that controls the strength of the regularization.

\section{Acknowledgements}

We are grateful to Guillaume Lajoie, James Murray, and Scott Owen for helpful feedback on the manuscript. We also wish to thank the Allen Institute for Brain Science founder, Paul G Allen, for his vision, encouragement and support. Helena Liu is supported by Natural the Science and Engineering Research Council (NSERC) Postgraduate Scholarships - Doctoral (NSERC PGS-D) program. This work was facilitated through the use of advanced computational, storage, and networking infrastructure provided by the Hyak supercomputer system at the University of Washington. 


\section{References}

[1] Marvin Minsky. "Steps toward artificial intelligence". In: Proceedings of the IRE 49.1 (1961), pp. 8-30.

[2] Pieter R. Roelfsema and Anthony Holtmaat. "Control of synaptic plasticity in deep cortical networks". In: Nature Reviews Neuroscience 19.3 (Feb. 2018), pp. 166-180. ISSN: 14710048. DOI: 10.1038/nrn.2018.6.

[3] Nan Rosemary Ke, Anirudh Goyal ALIAS PARTH GOYAL, Olexa Bilaniuk, Jonathan Binas, Michael C Mozer, Chris Pal, and Yoshua Bengio. "Sparse attentive backtracking: Temporal credit assignment through reminding". In: Advances in neural information processing systems. 2018, pp. 7640-7651.

[4] Blake A. Richards and Timothy P. Lillicrap. "Dendritic solutions to the credit assignment problem". In: Current Opinion in Neurobiology 54 (Feb. 2019), pp. 28-36. ISSN: 18736882. DOI: 10.1016/j.conb.2018.08.003.

[5] Yann Lecun, Yoshua Bengio, and Geoffrey Hinton. "Deep learning". In: Nature 521.7553 (May 2015), pp. 436444. ISSN: 14764687 . DOI: $10.1038 /$ nature14539.

[6] Ronald J. Williams and David Zipser. "A Learning Algorithm for Continually Running Fully Recurrent Neural Networks". In: Neural Computation 1.2 (June 1989), pp. 270-280. ISSN: 0899-7667. DOI: 10.1162/neco.1989. 1.2.270.

[7] Stephen J. Smith, Uygar Sümbül, Lucas T. Graybuck, Forrest Collman, Sharmishtaa Seshamani, Rohan Gala, Olga Gliko, Leila Elabbady, Jeremy A. Miller, Trygve E. Bakken, Jean Rossier, Zizhen Yao, Ed Lein, Hongkui Zeng, Bosiljka Tasic, and Michael Hawrylycz. "Single-cell transcriptomic evidence for dense intracortical neuropeptide networks". In: eLife 8 (Nov. 2019). ISSN: 2050084X. DOI: 10.7554/eLife.47889.

[8] Stephen J. Smith, Michael Hawrylycz, Jean Rossier, and Uygar Sümbül. "New light on cortical neuropeptides and synaptic network plasticity". In: Current Opinion in Neurobiology 63 (Aug. 2020), pp. 176-188. ISSN: 18736882. DOI: $10.1016 / \mathrm{j}$.conb.2020.04.002, arXiv: 2004.07975 .

[9] Bosiljka Tasic, Zizhen Yao, Lucas T. Graybuck, Kimberly A. Smith, Thuc Nghi Nguyen, Darren Bertagnolli, Jeff Goldy, Emma Garren, Michael N. Economo, Sarada Viswanathan, Osnat Penn, Trygve Bakken, Vilas Menon, Jeremy Miller, Olivia Fong, Karla E. Hirokawa, Kanan Lathia, Christine Rimorin, Michael Tieu, Rachael Larsen, Tamara Casper, Eliza Barkan, Matthew Kroll, Sheana Parry, Nadiya V. Shapovalova, Daniel Hirschstein, Julie Pendergraft, Heather A. Sullivan, Tae Kyung Kim, Aaron Szafer, Nick Dee, Peter Groblewski, Ian Wickersham, Ali Cetin, Julie A. Harris, Boaz P. Levi, Susan M. Sunkin, Linda Madisen, Tanya L. Daigle, Loren Looger, Amy Bernard, John Phillips, Ed Lein, Michael Hawrylycz, Karel Svoboda, Allan R. Jones, Christof Koch, and Hongkui Zeng. "Shared and distinct transcriptomic cell types across neocortical areas". In: Nature 563.7729 (Nov. 2018), pp. 72-78. ISSN: 14764687. DOI: 10.1038/s41586-018-0654-5.

[10] Nathan W Gouwens, Staci A Sorensen, Jim Berg, Changkyu Lee, Tim Jarsky, Jonathan Ting, Susan M Sunkin, David Feng, Costas A Anastassiou, Eliza Barkan, et al. "Classification of electrophysiological and morphological neuron types in the mouse visual cortex". In: Nature neuroscience 22.7 (2019), pp. 1182-1195.

[11] Ken Sugino, Erin Clark, Anton Schulmann, Yasuyuki Shima, Lihua Wang, David L Hunt, Bryan M Hooks, Dimitri Tränkner, Jayaram Chandrashekar, Serge Picard, et al. "Mapping the transcriptional diversity of genetically and anatomically defined cell populations in the mouse brain". In: Elife 8 (2019), e38619.

[12] Maria Antonietta Tosches and Gilles Laurent. "Evolution of neuronal identity in the cerebral cortex". In: Current opinion in neurobiology 56 (2019), pp. 199-208.

[13] Amit Zeisel, Hannah Hochgerner, Peter Lönnerberg, Anna Johnsson, Fatima Memic, Job Van Der Zwan, Martin Häring, Emelie Braun, Lars E Borm, Gioele La Manno, et al. "Molecular architecture of the mouse nervous system". In: Cell 174.4 (2018), pp. 999-1014.

[14] Hannah Bos, Anne-Marie Oswald, and Brent Doiron. "Untangling stability and gain modulation in cortical circuits with multiple interneuron classes". In: bioRxiv (2020).

[15] Yang Dan and Mu-Ming Poo. "Spike timing-dependent plasticity: from synapse to perception". In: Physiological reviews 86.3 (2006), pp. 1033-1048.

[16] Sen Song, Kenneth D Miller, and Larry F Abbott. "Competitive Hebbian learning through spike-timingdependent synaptic plasticity". In: Nature neuroscience 3.9 (2000), pp. 919-926.

[17] Michael A. Farries and Adrienne L. Fairhall. "Reinforcement Learning With Modulated Spike Timing-Dependent Synaptic Plasticity". In: Journal of Neurophysiology 98.6 (Dec. 2007), pp. 3648-3665. ISSN: 0022-3077. DOI: $10.1152 / j n .00364 .2007$. 
[18] Wolfram Schultz. "Dopamine reward prediction-error signalling: a two-component response". In: Nature Reviews Neuroscience 17.3 (2016), p. 183.

[19] Verena Pawlak, Jeffery R Wickens, Alfredo Kirkwood, and Jason ND Kerr. "Timing is not everything: neuromodulation opens the STDP gate". In: Frontiers in synaptic neuroscience 2 (2010), p. 146.

[20] Zuzanna Brzosko, Susanna B Mierau, and Ole Paulsen. "Neuromodulation of Spike-Timing-Dependent plasticity: past, present, and future". In: Neuron 103.4 (2019), pp. 563-581.

[21] Jeffrey C. Magee and Christine Grienberger. "Synaptic Plasticity Forms and Functions". In: Annual Review of Neuroscience 43.1 (July 2020), pp. 95-117. IsSN: 0147-006X. DOI: 10.1146/annurev-neuro-090919-022842

[22] Timothy P Lillicrap, Adam Santoro, Luke Marris, Colin J Akerman, and Geoffrey Hinton. "Backpropagation and the brain". In: Nature Reviews Neuroscience (2020), pp. 1-12.

[23] Alexandre Payeur, Jordan Guerguiev, Friedemann Zenke, Blake Richards, and Richard Naud. "Burst-dependent synaptic plasticity can coordinate learning in hierarchical circuits". In: bioRxiv (2020).

[24] Johnatan Aljadeff, James D'amour, Rachel E Field, Robert C Froemke, and Claudia Clopath. "Cortical credit assignment by Hebbian, neuromodulatory and inhibitory plasticity". In: arXiv preprint arXiv:1911.00307 (2019).

[25] Nicolas Frémaux and Wulfram Gerstner. "Neuromodulated spike-timing-dependent plasticity, and theory of three-factor learning rules". In: Frontiers in Neural Circuits 9.JAN2016 (Jan. 2015), p. 85. ISSN: 16625110. DOI: $10.3389 /$ fncir.2015.00085.

[26] Wulfram Gerstner, Marco Lehmann, Vasiliki Liakoni, Dane Corneil, and Johanni Brea. "Eligibility Traces and Plasticity on Behavioral Time Scales: Experimental Support of NeoHebbian Three-Factor Learning Rules". In: Frontiers in Neural Circuits 12 (July 2018), p. 53. ISSN: 16625110. DOI: 10.3389/fncir.2018.00053 arXiv: 1801.05219 .

[27] Răzvan V Florian. "Reinforcement learning through modulation of spike-timing-dependent synaptic plasticity". In: Neural computation 19.6 (2007), pp. 1468-1502.

[28] Wolfram Schultz. "Neuronal reward and decision signals: from theories to data". In: Physiological reviews 95.3 (2015), pp. 853-951.

[29] Demetrios K Vassilatis, John G Hohmann, Hongkui Zeng, Fusheng Li, Jane E Ranchalis, Marty T Mortrud, Analisa Brown, Stephanie S Rodriguez, John R Weller, Abbie C Wright, et al. "The G protein-coupled receptor repertoires of human and mouse". In: Proceedings of the National Academy of Sciences 100.8 (2003), pp. 4903-4908.

[30] Damien Jullié, Miriam Stoeber, Jean-Baptiste Sibarita, Hanna L Zieger, Thomas M Bartol, Seksiri Arttamangkul, Terrence J Sejnowski, Eric Hosy, and Mark von Zastrow. "A discrete presynaptic vesicle cycle for neuromodulator receptors". In: Neuron 105.4 (2020), pp. 663-677.

[31] Olivier Civelli. "Orphan GPCRs and neuromodulation". In: Neuron 76.1 (2012), pp. 12-21.

[32] Farzan Nadim and Dirk Bucher. "Neuromodulation of neurons and synapses". In: Current opinion in neurobiology 29 (2014), pp. 48-56.

[33] David A McCormick and Michael P Nusbaum. "Editorial overview: neuromodulation: tuning the properties of neurons, networks and behavior". In: Current opinion in neurobiology 29 (2014), p. iv.

[34] Stephen PH Alexander, Arthur Christopoulos, Anthony P Davenport, Eamonn Kelly, Alistair Mathie, John A Peters, Emma L Veale, Jane F Armstrong, Elena Faccenda, Simon D Harding, et al. "The Concise Guide to PHARMACOLOGY 2019/20: G protein-coupled receptors". In: British Journal of Pharmacology 176 (2019), S21-S141.

[35] Jean-Christophe Cassel. "The role of serotonin in learning and memory: a rich pallet of experimental studies". In: Handbook of Behavioral Neuroscience. Vol. 31. Elsevier, 2020, pp. 549-570.

[36] Amjad H Bazzari and H Rheinallt Parri. "Neuromodulators and long-term synaptic plasticity in learning and memory: A steered-glutamatergic perspective". In: Brain sciences 9.11 (2019), p. 300.

[37] Éva Borbély, Bálint Scheich, and Zsuzsanna Helyes. "Neuropeptides in learning and memory". In: Neuropeptides 47.6 (Dec. 2013), pp. 439-450. ISSN: 01434179. DOI: 10.1016/j.npep.2013.10.012.

[38] Stephen D Meriney and Erika Fanselow. "Synaptic Transmission". In: Academic Press, 2019. Chap. Neuropeptide Transmitters, pp. 421-434. 
[39] Anthony N. van den Pol. "Neuropeptide Transmission in Brain Circuits". In: Neuron 76.1 (Oct. 2012), pp. 98-115. ISSN: 08966273. DOI: 10.1016/j.neuron.2012.09.014.

[40] C. R. Gøtzsche and D. P.D. Woldbye. "The role of NPY in learning and memory". In: Neuropeptides 55 (Feb. 2016), pp. 79-89. ISSN: 15322785. DOI: 10.1016/j .npep.2015.09.010.

[41] Sarah Melzer, Elena Newmark, Grace Or Mizuno, Minsuk Hyun, Adrienne C Philson, Eleonora Quiroli, Beatrice Righetti, Malika R Gregory, Kee Wui Huang, James Levasseur, et al. "Bombesin-like peptide recruits disinhibitory cortical circuits and enhances fear memories". In: Available at SSRN 3724673 (2020).

[42] Gáspár Jékely. "Global view of the evolution and diversity of metazoan neuropeptide signaling". In: Proceedings of the National Academy of Sciences 110.21 (2013), pp. 8702-8707.

[43] J Peter H Burbach. "What are neuropeptides?" In: Neuropeptides. Springer, 2011, pp. 1-36.

[44] Dick R Nässel. "Neuropeptide signaling near and far: how localized and timed is the action of neuropeptides in brain circuits?" In: Invertebrate neuroscience 9.2 (2009), p. 57.

[45] Gareth Leng and Mike Ludwig. "Neurotransmitters and peptides: whispered secrets and public announcements". In: The Journal of physiology 586.23 (2008), pp. 5625-5632.

[46] Valerio Mante, David Sussillo, Krishna V. Shenoy, and William T. Newsome. "Context-dependent computation by recurrent dynamics in prefrontal cortex". In: Nature 503.7474 (Nov. 2013), pp. 78-84. ISSN: 00280836. DOI: 10.1038/nature12742.

[47] James M. Murray. "Local online learning in recurrent networks with random feedback". In: eLife 8 (May 2019). ISSN: 2050084X. DOI: 10.7554 /eLife.43299.

[48] Guillaume Bellec, Franz Scherr, Anand Subramoney, Elias Hajek, Darjan Salaj, Robert Legenstein, and Wolfgang Maass. "A solution to the learning dilemma for recurrent networks of spiking neurons". In: Nature Communications 11.1 (Dec. 2020), pp. 1-15. IsSN: 20411723. DOI: 10.1038/s41467-020-17236-y.

[49] Martin Boerlin, Christian K. Machens, and Sophie Denève. "Predictive Coding of Dynamical Variables in Balanced Spiking Networks". In: PLoS Computational Biology 9.11 (Nov. 2013), p. 1003258. Issn: 1553734X. DOI: $10.1371 /$ journal.pcbi.1003258

[50] Eric Hunsberger and Chris Eliasmith. "Spiking deep networks with LIF neurons". In: arXiv preprint arXiv:1510.08829 (2015).

[51] Robert Kim, Yinghao Li, and Terrence J. Sejnowski. "Simple framework for constructing functional spiking recurrent neural networks". In: Proceedings of the National Academy of Sciences of the United States of America 116.45 (Nov. 2019), pp. 22811-22820. ISSN: 10916490. DOI: 10.1073/pnas.1905926116.

[52] Robert Kim and Terrence J Sejnowski. "Strong inhibitory signaling underlies stable temporal dynamics and working memory in spiking neural networks". In: bioRxiv (2020).

[53] Kaushik Roy, Akhilesh Jaiswal, and Priyadarshini Panda. "Towards spike-based machine intelligence with neuromorphic computing". In: Nature 575.7784 (2019), pp. 607-617.

[54] Jacob Menick, Erich Elsen, Utku Evci, Simon Osindero, Karen Simonyan, and Alex Graves. "A Practical Sparse Approximation for Real Time Recurrent Learning". In: arXiv preprint arXiv:2006.07232 (2020).

[55] Friedemann Zenke and Emre O Neftci. "Brain-Inspired Learning on Neuromorphic Substrates". In: arXiv preprint arXiv:2010.11931 (2020).

[56] Stijn Cassenaer and Gilles Laurent. "Conditional modulation of spike-timing-dependent plasticity for olfactory learning”. In: Nature 482.7383 (Feb. 2012), pp. 47-51. ISSN: 14764687. DOI: 10.1038/nature10776.

[57] Sho Yagishita, Akiko Hayashi-Takagi, Graham C.R. Ellis-Davies, Hidetoshi Urakubo, Shin Ishii, and Haruo Kasai. "A critical time window for dopamine actions on the structural plasticity of dendritic spines". In: Science 345.6204 (Sept. 2014), pp. 1616-1620. ISSN: 10959203. DOI: 10.1126/science.1255514.

[58] Marco P Lehmann, He A Xu, Vasiliki Liakoni, Michael H Herzog, Wulfram Gerstner, and Kerstin Preuschoff. "One-shot learning and behavioral eligibility traces in sequential decision making". In: Elife 8 (2019), e47463.

[59] Aparna Suvrathan. "Beyond STDP - towards diverse and functionally relevant plasticity rules". In: Current Opinion in Neurobiology 54 (Feb. 2019), pp. 12-19. ISSN: 18736882. DOI: 10.1016/j.conb.2018.06.011.

[60] Valentino Braitenberg and Almut Schüz. Cortex: statistics and geometry of neuronal connectivity. Springer Science \& Business Media, 2013. 
[61] Stephanie C. Seeman, Luke Campagnola, Pasha A. Davoudian, Alex Hoggarth, Travis A. Hage, Alice BosmaMoody, Christopher A. Baker, Jung Hoon Lee, Stefan Mihalas, Corinne Teeter, Andrew L. Ko, Jeffrey G. Ojemann, Ryder P. Gwinn, Daniel L. Silbergeld, Charles Cobbs, John Phillips, Ed Lein, Gabe Murphy, Christof Koch, Hongkui Zeng, and Tim Jarsky. "Sparse recurrent excitatory connectivity in the microcircuit of the adult mouse and human cortex". In: eLife 7 (Sept. 2018). ISSN: 2050084X. DOI: 10.7554/eLife.37349

[62] Guillaume Bellec, David Kappel, Wolfgang Maass, and Robert Legenstein. "Deep rewiring: Training very sparse deep networks". In: arXiv preprint arXiv:1711.05136 (2017).

[63] Guillaume Bellec, Darjan Salaj, Anand Subramoney, Robert Legenstein, and Wolfgang Maass. "Long shortterm memory and learning-to-learn in networks of spiking neurons". In: 32nd Conference on Neural Information Processing Systems. 2018, pp. 787-797.

[64] Timothy P Lillicrap, Daniel Cownden, Douglas B Tweed, and Colin J Akerman. "Random synaptic feedback weights support error backpropagation for deep learning". In: Nature communications 7.1 (2016), pp. 1-10.

[65] Wilten Nicola and Claudia Clopath. "Supervised learning in spiking neural networks with FORCE training". In: Nature Communications 8.1 (Dec. 2017), pp. 1-15. ISSN: 20411723. DOI: 10.1038/s41467-017-01827-3. arXiv: 1609.02545 .

[66] Peter Dayan and Laurence F Abbott. Theoretical neuroscience: computational and mathematical modeling of neural systems. Computational Neuroscience Series, 2001.

[67] William R Softky and Christof Koch. "The highly irregular firing of cortical cells is inconsistent with temporal integration of random EPSPs". In: Journal of Neuroscience 13.1 (1993), pp. 334-350.

[68] Travis Meyer, Xue Lian Qi, Terrence R. Stanford, and Christos Constantinidis. "Stimulus selectivity in dorsal and ventral prefrontal cortex after training in working memory tasks". In: Journal of Neuroscience 31.17 (Apr. 2011), pp. 6266-6276. ISSN: 02706474. DOI: 10.1523/JNEUROSCI.6798-10.2011.

[69] Ben Engelhard, Joel Finkelstein, Julia Cox, Weston Fleming, Hee Jae Jang, Sharon Ornelas, Sue Ann Koay, Stephan Y. Thiberge, Nathaniel D. Daw, David W. Tank, and Ilana B. Witten. "Specialized coding of sensory, motor and cognitive variables in VTA dopamine neurons". In: Nature 570.7762 (June 2019), pp. 509-513. ISSN: 14764687. DOI: 10.1038/s41586-019-1261-9.

[70] Ari S. Morcos and Christopher D. Harvey. "History-dependent variability in population dynamics during evidence accumulation in cortex". In: Nature Neuroscience 19.12 (Dec. 2016), pp. 1672-1681. ISSN: 15461726. DOI: $10.1038 / \mathrm{nn} .4403$

[71] Nicolas X. Tritsch and Bernardo L. Sabatini. "Dopaminergic Modulation of Synaptic Transmission in Cortex and Striatum". In: Neuron 76.1 (Oct. 2012), pp. 33-50. ISSN: 08966273. DOI: 10.1016/j.neuron.2012.09.023

[72] Tommaso Patriarchi, Jounhong Ryan Cho, Katharina Merten, Mark W Howe, Aaron Marley, Wei-Hong Xiong, Robert W Folk, Gerard Joey Broussard, Ruqiang Liang, Min Jee Jang, et al. "Ultrafast neuronal imaging of dopamine dynamics with designed genetically encoded sensors". In: Science 360.6396 (2018).

[73] Ao Dong, Kaikai He, Barna Dudok, Jordan S Farrell, Wuqiang Guan, Daniel J Liput, Henry L Puhl, Ruyi Cai, Jiali Duan, Eddy Albarran, et al. "A fluorescent sensor for spatiotemporally resolved endocannabinoid dynamics in vitro and in vivo". In: bioRxiv (2020).

[74] Gord Fishell and Adam Kepecs. "Interneuron types as attractors and controllers". In: Annual review of neuroscience 43 (2019).

[75] Ozgun Gokce, Geoffrey M Stanley, Barbara Treutlein, Norma F Neff, J Gray Camp, Robert C Malenka, Patrick E Rothwell, Marc V Fuccillo, Thomas C Südhof, and Stephen R Quake. "Cellular taxonomy of the mouse striatum as revealed by single-cell RNA-seq". In: Cell reports 16.4 (2016), pp. 1126-1137.

[76] Rebecca D Hodge, Trygve E Bakken, Jeremy A Miller, Kimberly A Smith, Eliza R Barkan, Lucas T Graybuck, Jennie L Close, Brian Long, Nelson Johansen, Osnat Penn, et al. "Conserved cell types with divergent features in human versus mouse cortex". In: Nature 573.7772 (2019), pp. 61-68.

[77] Sinisa Hrvatin, Daniel R Hochbaum, M Aurel Nagy, Marcelo Cicconet, Keiramarie Robertson, Lucas Cheadle, Rapolas Zilionis, Alex Ratner, Rebeca Borges-Monroy, Allon M Klein, et al. "Single-cell analysis of experiencedependent transcriptomic states in the mouse visual cortex". In: Nature neuroscience 21.1 (2018), pp. 120129.

[78] Z Josh Huang and Anirban Paul. "The diversity of GABAergic neurons and neural communication elements". In: Nature Reviews Neuroscience 20.9 (2019), pp. 563-572. 
[79] Daniel J Miller, Aparna Bhaduri, Nenad Sestan, and Arnold Kriegstein. "Shared and derived features of cellular diversity in the human cerebral cortex". In: Current opinion in neurobiology 56 (2019), pp. 117-124.

[80] Blake A Richards, Timothy P Lillicrap, Philippe Beaudoin, Yoshua Bengio, Rafal Bogacz, Amelia Christensen, Claudia Clopath, Rui Ponte Costa, Archy de Berker, Surya Ganguli, et al. "A deep learning framework for neuroscience". In: Nature neuroscience 22.11 (2019), pp. 1761-1770.

[81] Drew Linsley, Alekh Karkada Ashok, Lakshmi Narasimhan Govindarajan, Rex Liu, and Thomas Serre. "Stable and expressive recurrent vision models". In: arXiv preprint arXiv:2005.11362 (2020).

[82] Owen Marschall, Kyunghyun Cho, and Cristina Savin. "A unified framework of online learning algorithms for training recurrent neural networks". In: Journal of Machine Learning Research 21.135 (2020), pp. 1-34.

[83] Asier Mujika, Florian Meier, and Angelika Steger. "Approximating Real-Time Recurrent Learning with Random Kronecker Factors". In: 32nd Conference on Neural Information Processing Systems. 2018, pp. 65946603.

[84] Corentin Tallec and Yann Ollivier. "Unbiased Online Recurrent Optimization". In: ICLR. Feb. 2018.

[85] Christopher Roth, Ingmar Kanitscheider, and Ila Fiete. "Kernel RNN Learning (KERNL)". In: ICLR. Sept. 2019.

[86] Dongsung Huh and Terrence J Sejnowski. "Gradient Descent for Spiking Neural Networks". In: 32nd Conference on Neural Information Processing Systems. 2018, pp. 1433-1443.

[87] Emre O. Neftci, Hesham Mostafa, and Friedemann Zenke. "Surrogate Gradient Learning in Spiking Neural Networks: Bringing the Power of Gradient-based optimization to spiking neural networks". In: IEEE Signal Processing Magazine 36.6 (Nov. 2019), pp. 51-63. ISSN: 15580792. DOI: 10.1109/MSP.2019.2931595.

[88] Magdalena Sanhueza and John Lisman. "The CaMKII/NMDAR complex as a molecular memory". In: Molecular Brain 6.1 (Feb. 2013), p. 10. ISSN: 17566606. DOI: 10.1186/1756-6606-6-10.

[89] Amirsaman Sajad, David C. Godlove, and Jeffrey D. Schall. "Cortical microcircuitry of performance monitoring". In: Nature Neuroscience 22.2 (Feb. 2019), pp. 265-274. ISSN: 15461726. DOI: 10.1038/s41593-0180309-8.

[90] Trevor J. Hamilton, Sara Xapelli, Sheldon D. Michaelson, Matthew E. Larkum, and William F. Colmers. "Modulation of distal calcium electrogenesis by neuropeptide Y1 receptors inhibits neocortical long-term depression". In: Journal of Neuroscience 33.27 (July 2013), pp. 11184-11193. ISSN: 02706474. DOI: 10.1523/ JNEUROSCI .5595-12.2013,

[91] Hui Lu, Hyungju Park, and Mu-Ming Poo. "Spike-timing-dependent BDNF secretion and synaptic plasticity". In: Philosophical Transactions of the Royal Society B: Biological Sciences 369.1633 (2014), p. 20130132.

[92] Elissa J Donzis and Natalie C Tronson. "Modulation of learning and memory by cytokines: signaling mechanisms and long term consequences". In: Neurobiology of learning and memory 115 (2014), pp. 68-77.

[93] Jeffrey J Liu, Kirti Sharma, Luca Zangrandi, Chongguang Chen, Sean J Humphrey, Yi-Ting Chiu, Mariana Spetea, Lee-Yuan Liu-Chen, Christoph Schwarzer, and Matthias Mann. "In vivo brain GPCR signaling elucidated by phosphoproteomics". In: Science 360.6395 (2018).

[94] Skylar M Spangler and Michael R Bruchas. "Optogenetic approaches for dissecting neuromodulation and GPCR signaling in neural circuits". In: Current opinion in pharmacology 32 (2017), pp. 56-70.

[95] Corinne Teeter, Ramakrishnan Iyer, Vilas Menon, Nathan Gouwens, David Feng, Jim Berg, Aaron Szafer, Nicholas Cain, Hongkui Zeng, Michael Hawrylycz, et al. "Generalized leaky integrate-and-fire models classify multiple neuron types". In: Nature communications 9.1 (2018), pp. 1-15.

[96] Steven K. Esser, Paul A. Merolla, John V. Arthur, Andrew S. Cassidy, Rathinakumar Appuswamy, Alexander Andreopoulos, David J. Berg, Jeffrey L. McKinstry, Timothy Melano, Davis R. Barch, Carmelo Di Nolfo, Pallab Datta, Arnon Amir, Brian Taba, Myron D. Flickner, and Dharmendra S. Modha. "Convolutional networks for fast, energy-efficient neuromorphic computing". In: Proceedings of the National Academy of Sciences of the United States of America 113.41 (Oct. 2016), pp. 11441-11446. ISSN: 10916490. DOI: 10.1073/pnas.1604850113, arXiv: 1603.08270.

[97] Nal Kalchbrenner, Erich Elsen, Karen Simonyan, Seb Noury, Norman Casagrande, Edward Lockhart, Florian Stimberg, Aaron van den Oord, Sander Dieleman, and Koray Kavukcuoglu. "Efficient neural audio synthesis". In: arXiv preprint arXiv:1802.08435 (2018). 
[98] Sharan Narang, Erich Elsen, Gregory Diamos, and Shubho Sengupta. "Exploring sparsity in recurrent neural networks". In: arXiv preprint arXiv:1704.05119 (2017).

[99] Peter D. Welch. "The Use of Fast Fourier Transform for the Estimation of Power Spectra: A Method Based on Time Averaging Over Short, Modified Periodograms". In: IEEE Transactions on Audio and Electroacoustics 15.2 (1967), pp. 70-73. ISSN: 00189278. DOI: 10.1109/TAU.1967.1161901

[100] Diederik P. Kingma and Jimmy Lei Ba. "Adam: A method for stochastic optimization". In: ICLR. International Conference on Learning Representations, ICLR, Dec. 2015. arXiv: 1412.6980 


\section{Supplementary Materials}

\section{Supplementary Figures}

a

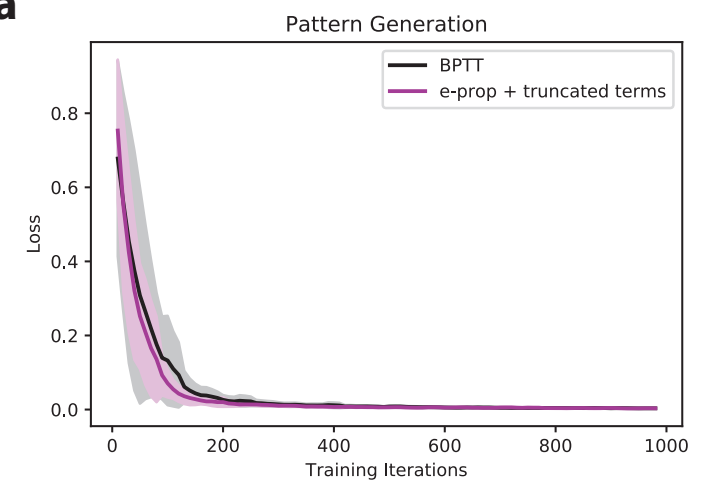

b

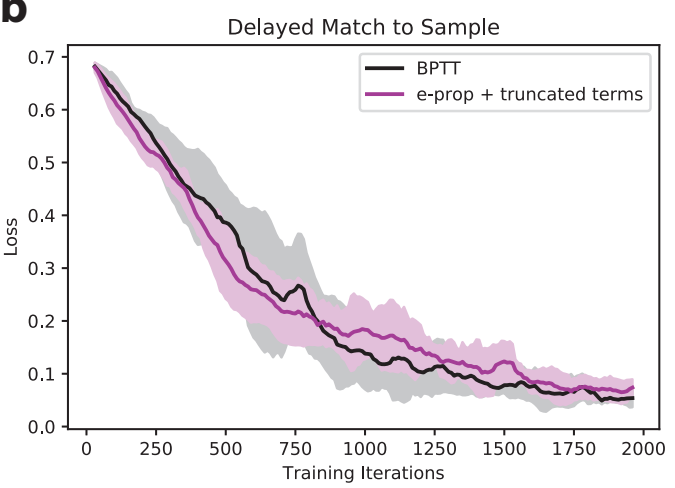

C

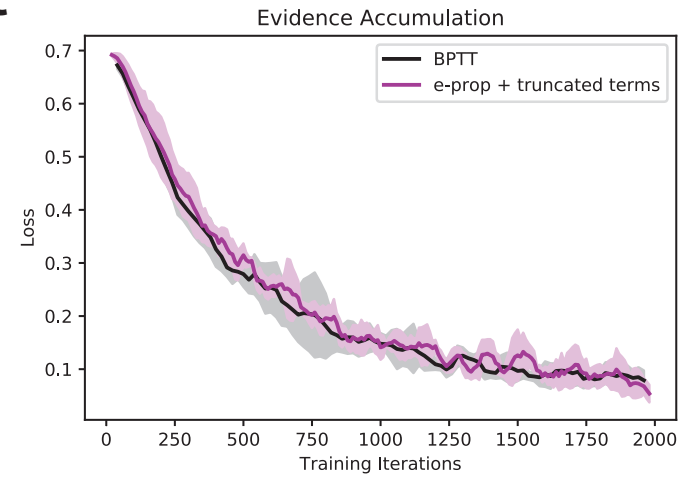

Figure S1: Checking e-prop implementation - recovering ignored terms recovers the performance of BPTT. As a sanity check, learning curves are plotted for e-prop plus all the truncated terms (see Eq. 1) to verify that the resulting learning rule recovers the performance of BPTT. The check is applied to a) pattern generation, b) delayed match to sample and c) evidence accumulation tasks. Solid lines show the mean averaged across five runs and shaded regions show the standard deviation. For all tasks, the learning curves do not differ significantly, suggesting the e-prop implementation is accurate. 
bioRxiv preprint doi: https://doi.org/10.1101/2020.11.22.393504; this version posted March 6, 2021. The copyright holder for this preprint (which was not certified by peer review) is the author/funder, who has granted bioRxiv a license to display the preprint in perpetuity. It is made available under aCC-BY-NC 4.0 International license.

a

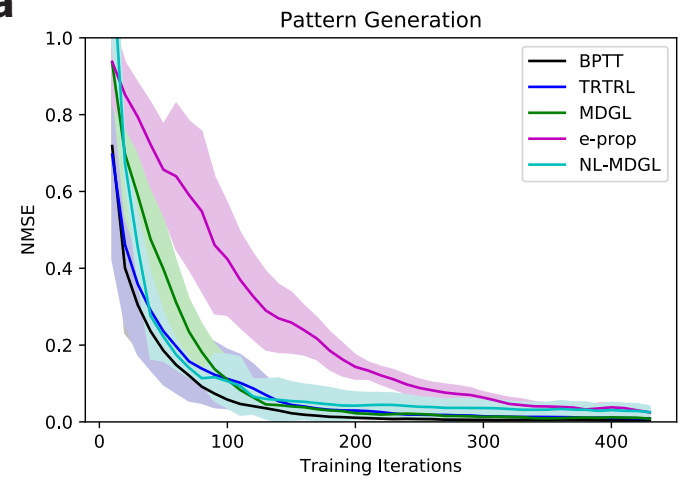

C

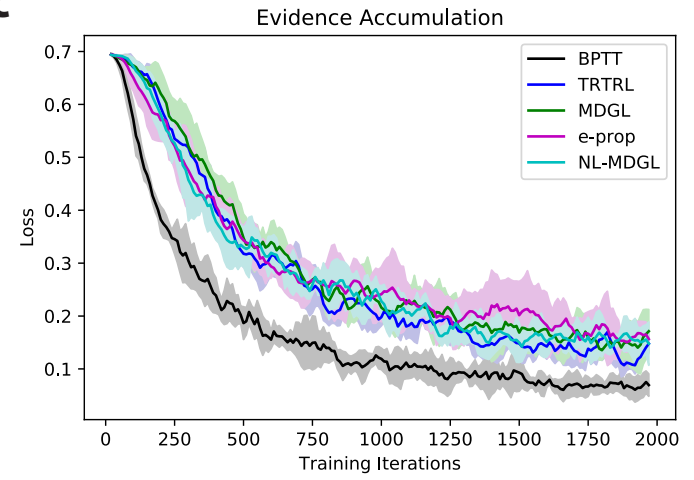

b

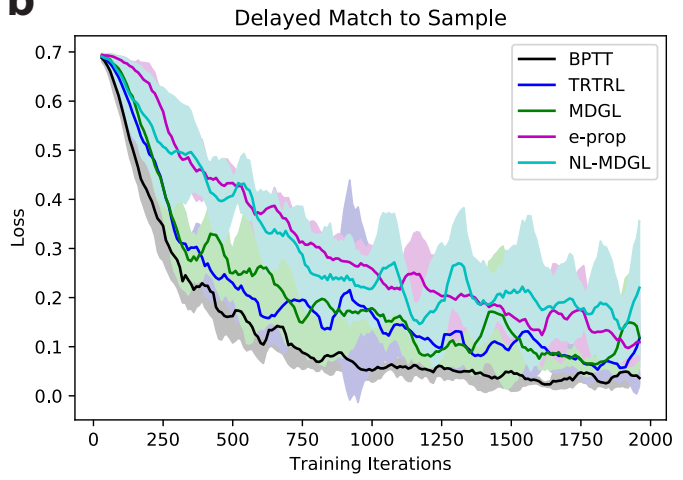

Figure S2: Learning performance of RSNNs with $30 \%$ connection probability. Learning curves for training methods in Figure $2 \mathrm{a}$ are illustrated for the following tasks: a) pattern generation, b) delayed match to sample and c) evidence accumulation tasks. The learning curve gap here between e-prop and MDGL is narrower than that of the network with $10 \%$ connection probability shown in the main text. This suggests that MDGL is more helpful under sparse scenarios. As noted in the main text, connection sparsity is widely observed in the brain 60 and has various computational advantages 97,98 . 
bioRxiv preprint doi: https://doi.org/10.1101/2020.11.22.393504; this version posted March 6, 2021. The copyright holder for this preprint (which was not certified by peer review) is the author/funder, who has granted bioRxiv a license to display the preprint in perpetuity. It is made available under aCC-BY-NC 4.0 International license.

a

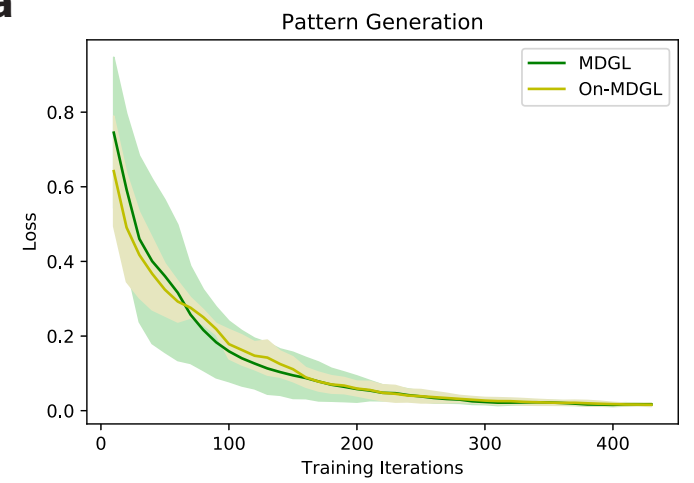

C

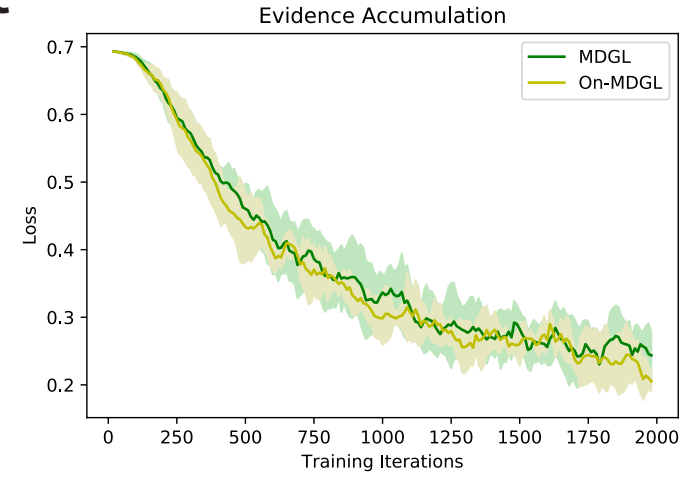

b

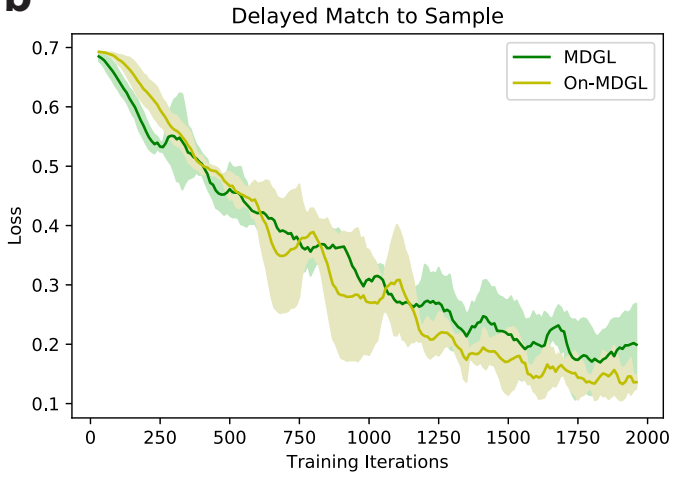

Figure S3: No significant degradation in performance observed for the online approximation of MDGL in Eq. S3 For outputs with a leak term defined in Eq. M2 $\frac{\partial E}{\partial z_{j, t}}$ depends on future errors and an approximation is introduced in Eq. S3 for online implementation of MDGL. To check if this approximation leads to significant degradation in performance, learning curves are plotted for a) pattern generation, b) delayed match to sample and c) evidence accumulation tasks. For all tasks, there is no significant deviation in learning curves between MDGL and the online approximation (On-MDGL). 
bioRxiv preprint doi: https://doi.org/10.1101/2020.11.22.393504; this version posted March 6, 2021. The copyright holder for this preprint (which was not certified by peer review) is the author/funder, who has granted bioRxiv a license to display the preprint in perpetuity. It is made available under aCC-BY-NC 4.0 International license.
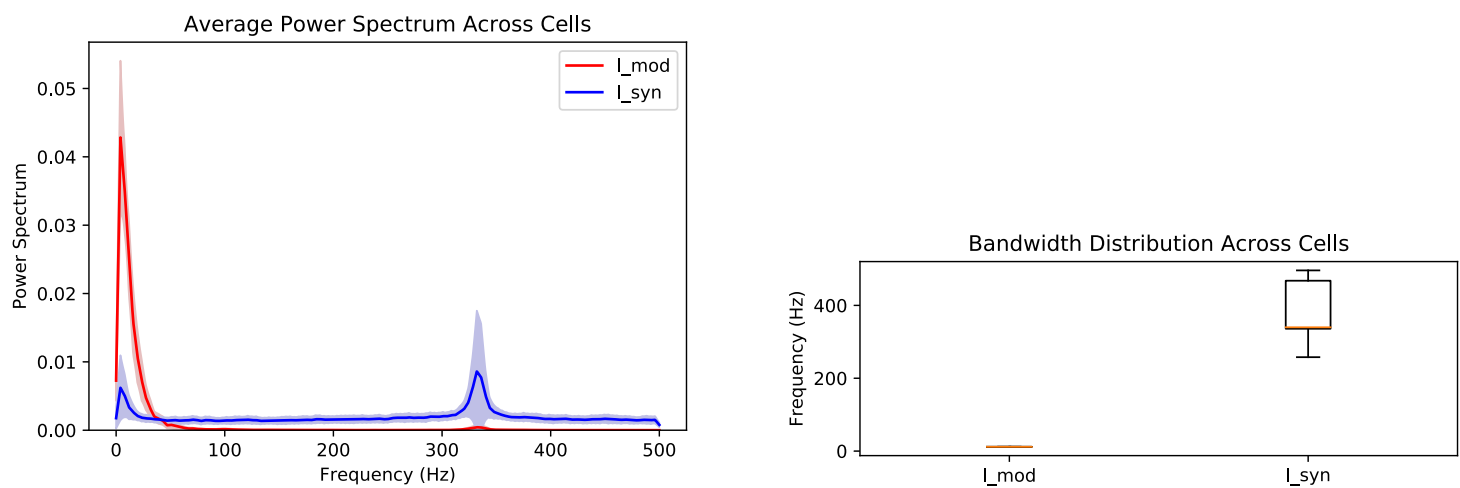

Figure S4: Slowness in modulatory signaling for the online approximation of MDGL. We repeat the spectral analysis in Figure 7 but for the online implementation of local modulatory input in Eq. S3 i.e. $I_{m o d, p}:=\sum_{\alpha \in C} w_{\alpha \beta} \sum_{j \in \alpha, p \rightarrow j} \bar{a}_{j, t}$ with $\bar{a}_{j, t}$ defined $\mathrm{in}$ Eq. S3 The observations here match those of Figure 7. where modulatory input is significantly slower than synaptic input. We note that the analysis here is done on the pattern generation task only, because for the other two tasks, the error signal is not available until the end of the trial, making the modulatory input too short (see Eq. S3 for any meaningful spectral analysis. We expect this "slowness" of modulatory signaling to generalize as the modulatory input is a weighted summation of slow changing leaky outputs and low-pass filtered activity $h_{j, t}$. 
bioRxiv preprint doi: https://doi.org/10.1101/2020.11.22.393504; this version posted March 6, 2021. The copyright holder for this preprint (which was not certified by peer review) is the author/funder, who has granted bioRxiv a license to display the preprint in perpetuity. It is made available under aCC-BY-NC 4.0 International license.
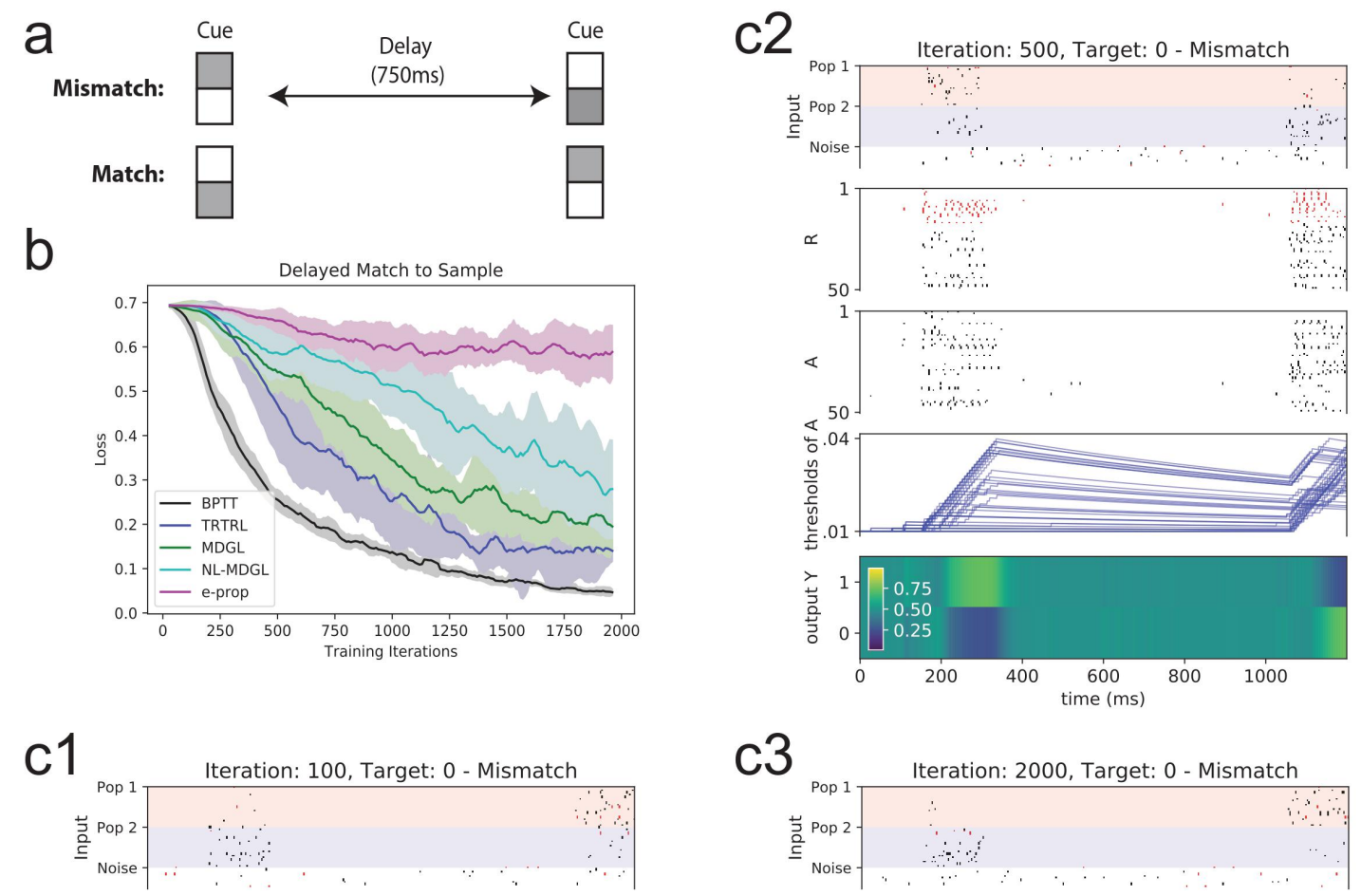

c3
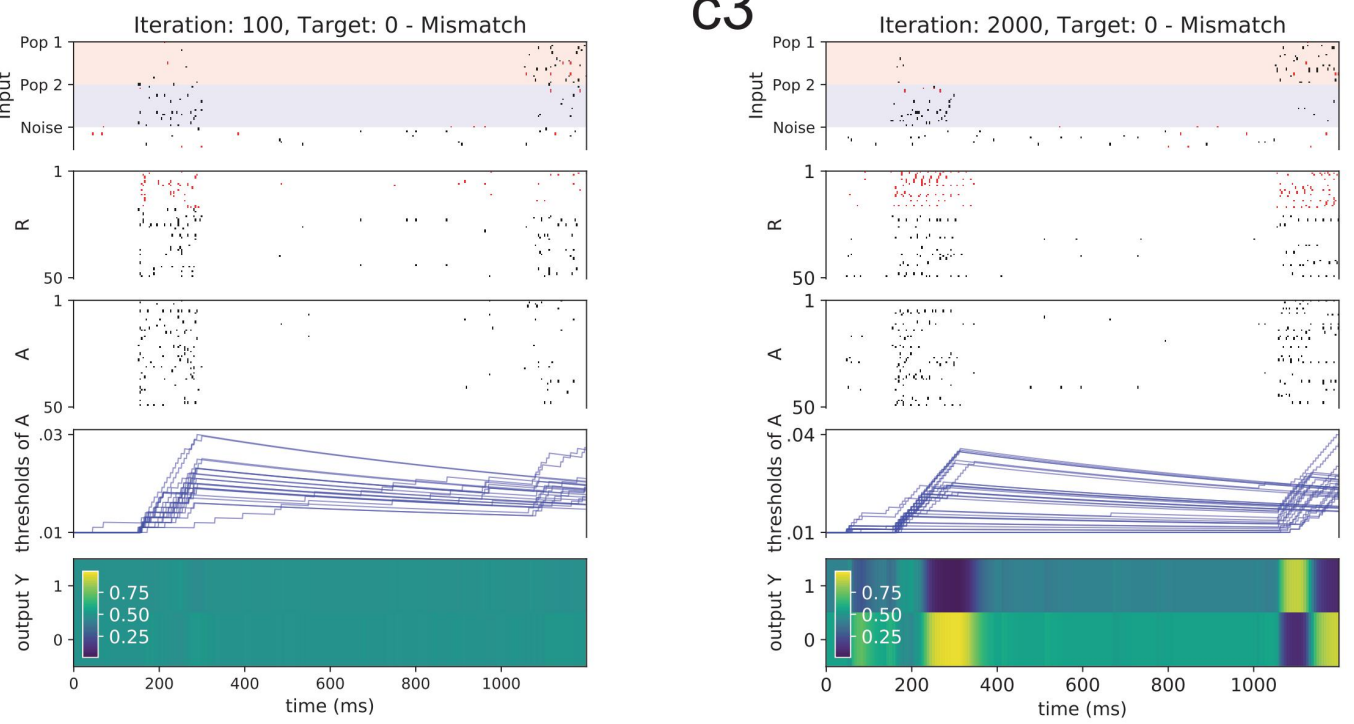

Figure S5: Similar observations for alternative task parameters in the task of Figure 5 The delayed match-to-sample task in Figure 5 is repeated here, but with nonzero firing rates for the second cue alternative. As before, two input populations take on two different firing statistics to represent the two cue alternatives, and the agent is tasked with determining if the cue presented before and after the delay period correspond to the same cue alternative. Here, the rates of these two populations provided in Supplementary Note 3 The plotting conventions here are the same as those of Figure 5 except that a larger network is used (see Supplementary Note 3 ) so for better readability, only 50 units are selected for the raster plots. The same conclusion as Figure 5 is observed here: comparing the performance of e-prop with the MDGL method suggests that the addition of cell-type-specific modulatory signals expedites the learning curve; the network makes the correct prediction with greater confidence as training progresses. 
bioRxiv preprint doi: https://doi.org/10.1101/2020.11.22.393504; this version posted March 6, 2021. The copyright holder for this preprint (which was not certified by peer review) is the author/funder, who has granted bioRxiv a license to display the preprint in perpetuity. It is made available under aCC-BY-NC 4.0 International license.
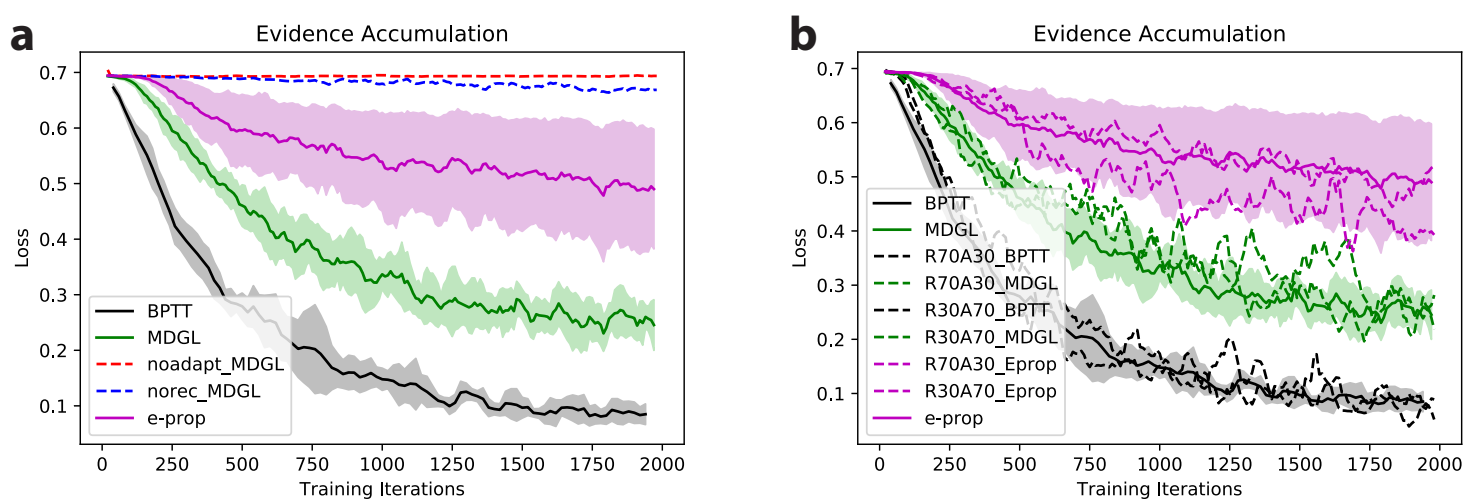

Figure S6: Threshold adaptation analysis for the evidence accumulation task in Figure 6 a) Both threshold adaptation and recurrence are needed for successful completion of the task. MDGL trained on a network without ALIF cells (red) or with recurrent connections removed (blue) shows little decrease in loss function. b) Simulating with 70 LIF to 30 ALIF cells (R70A30) as well as 30 LIF to 70 ALIF cells (R30A79) led to similar ordering of performance for different learning methods as the default (50 LIF to 50 ALIF cells). Here, dashed lines represent individual runs. 
bioRxiv preprint doi: https://doi.org/10.1101/2020.11.22.393504; this version posted March 6, 2021. The copyright holder for this preprint (which was not certified by peer review) is the author/funder, who has granted bioRxiv a license to display the preprint in perpetuity. It is made available under aCC-BY-NC 4.0 International license.

ai

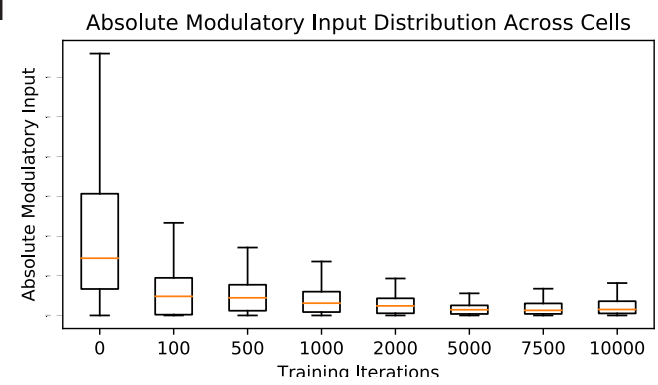

b

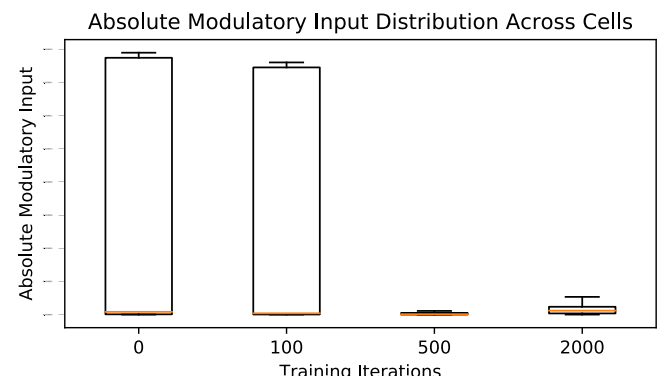

aii

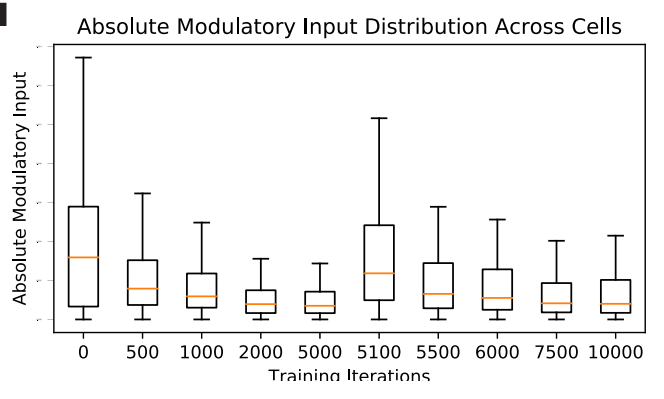

C

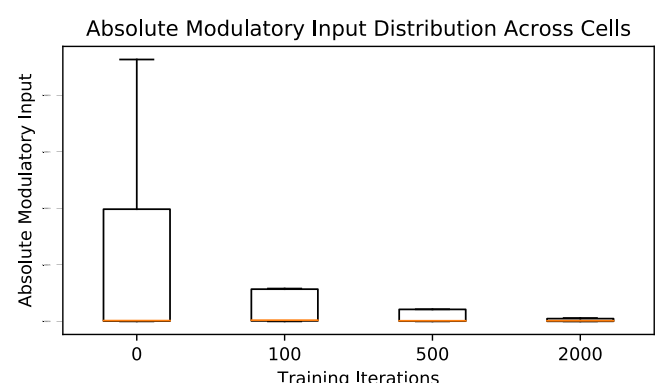

Figure S7: Cell-type-specific modulatory signaling level decreases over training iterations. Box plots for absolute celltype-specific modulatory input distribution across cells show that modulatory signalling drops over training iterations for ai) pattern generation, b) delayed match-to-sample and c) evidence accumulation tasks. In aii), the target for the pattern generation task was changed after 5000 iterations, which resulted in a rapid increase in modulatory input immediately after the change, and a progressive decrease as training continues. Here, absolute modulatory input for cell $p$ is defined as $\sum_{\alpha \in C}\left|w_{\alpha \beta}\right| \sum_{j \in \alpha, p \rightarrow j}\left|\bar{a}_{j, t}\right|\left(\bar{a}_{j, t}\right.$ is defined in Eq. S3, where the absolute value is taken because we are interested in the magnitude of the signaling level. 
bioRxiv preprint doi: https://doi.org/10.1101/2020.11.22.393504; this version posted March 6, 2021. The copyright holder for this preprint (which was not certified by peer review) is the author/funder, who has granted bioRxiv a license to display the preprint in perpetuity. It is made available under aCC-BY-NC 4.0 International license.

a

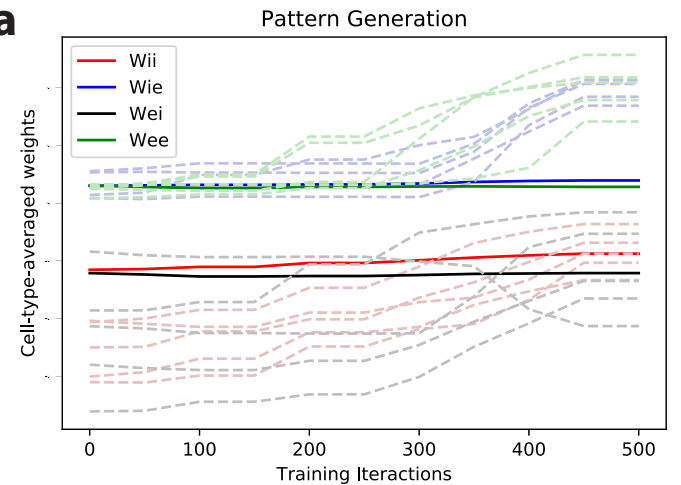

C

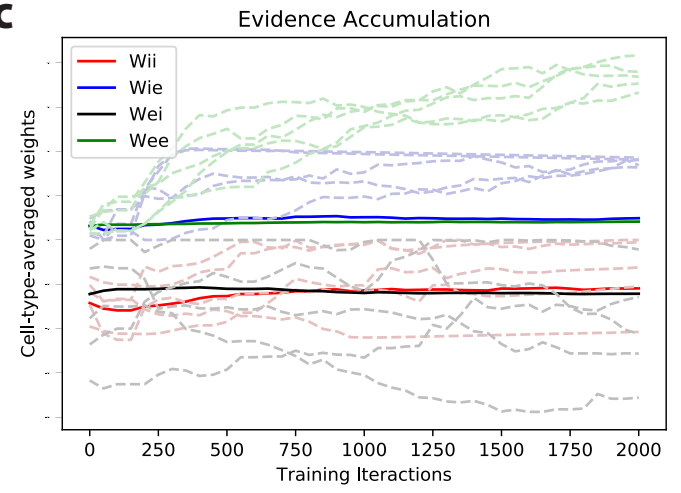

b

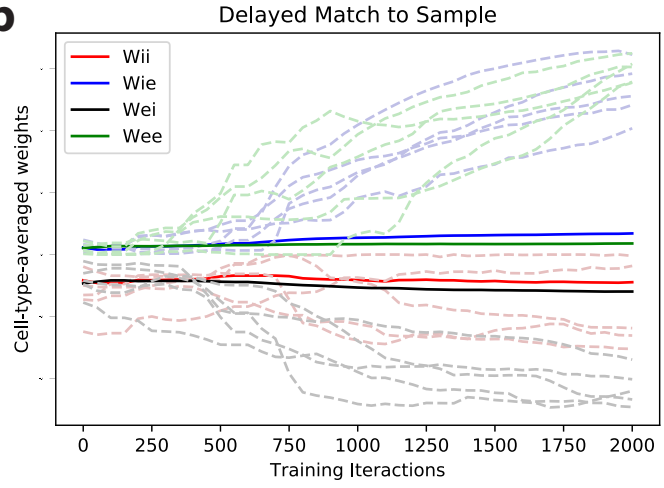

Figure S8: Cell-type-specific signaling gain drifts slowly over training. The four cell-type-specific signaling gains for MDGL with two cell-types, i.e. $w_{\alpha \beta}$ in Eq. 4 and Eq. 5 with $\alpha, \beta \in\{E, I\}$, are illustrated in solid lines for the three tasks investigated. Several sample individual weights with large changes are illustrated in faint dashed lines. Because we implemented cell-type-specific signaling gain using weight averages, it is not surprising that they drift over training as weights adapt. This drift, however, is slow compared to how fast some individual weights can change. 
bioRxiv preprint doi: https://doi.org/10.1101/2020.11.22.393504; this version posted March 6, 2021. The copyright holder for this preprint (which was not certified by peer review) is the author/funder, who has granted bioRxiv a license to display the preprint in perpetuity. It is made available under aCC-BY-NC 4.0 International license.

a

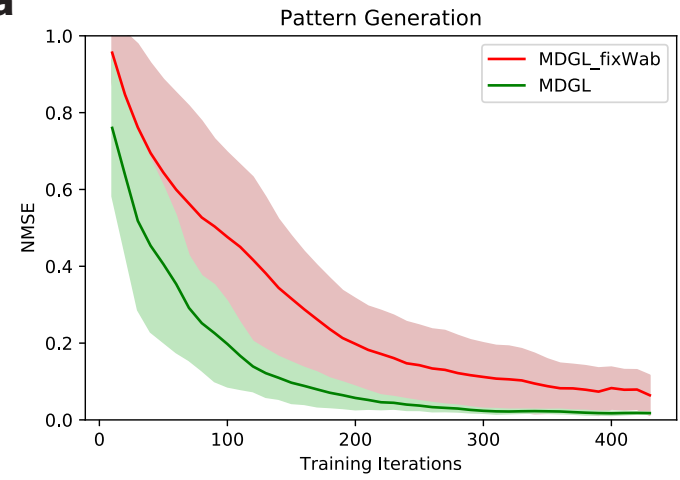

C

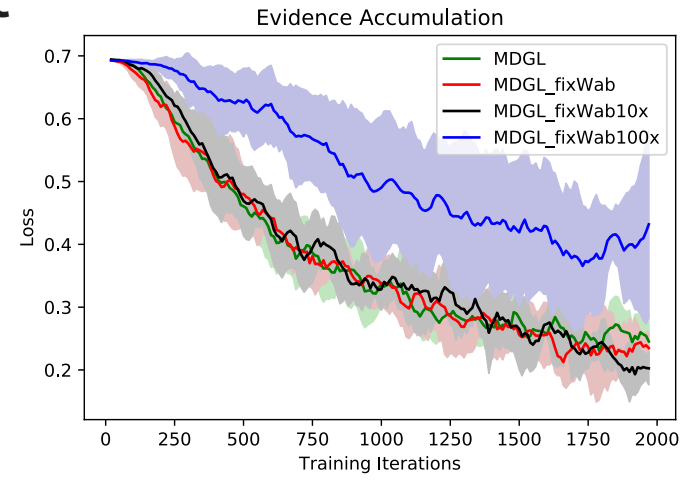

b

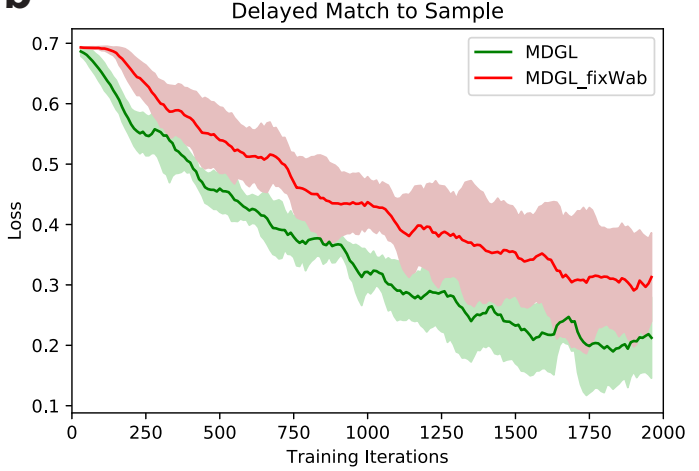

Figure S9: Comparing the learning curves for default MDGL versus MDGL using fixed cell-type-specific gain $w_{\alpha \beta}$. This comparison is done for a) pattern generation, b) delayed match-to-sample and c) evidence accumulation tasks. Here, the magnitude of each $w_{\alpha \beta}$ (for $\alpha \in\{I, E\}, \beta \in\{I, E\}$ ) is randomly generated using a Gaussian random variable with zero mean and variance of $\frac{1}{\sqrt{N}}$ upon each initialization and fixed throughout the training. The sign of $w_{\alpha \beta}$ is taken to be the sign of its presynaptic group. We observe degradation in performance for the pattern generation task and delayed match-to-sample tasks using this fixed random $w_{\alpha \beta}$ (labeled as MDGL fixWab in each panel), but not for evidence accumulation task. Multiplying this random $w_{\alpha \beta}$ by a factor of 100 (labeled as MDGL_fixWab100x) pushes the network outside of an efficient operating range for the evidence accumulation task, but the training for this task appears to be robust even when the randomly generated $w_{\alpha \beta}$ is multiplied by a factor of 10 (labeled as MDGL_fixWab10x). This suggests that different tasks exhibit different degree of tolerance to deviations in $w_{\alpha \beta}$. 
bioRxiv preprint doi: https://doi.org/10.1101/2020.11.22.393504; this version posted March 6, 2021. The copyright holder for this preprint (which was not certified by peer review) is the author/funder, who has granted bioRxiv a license to display the preprint in perpetuity. It is made available under aCC-BY-NC 4.0 International license.

a
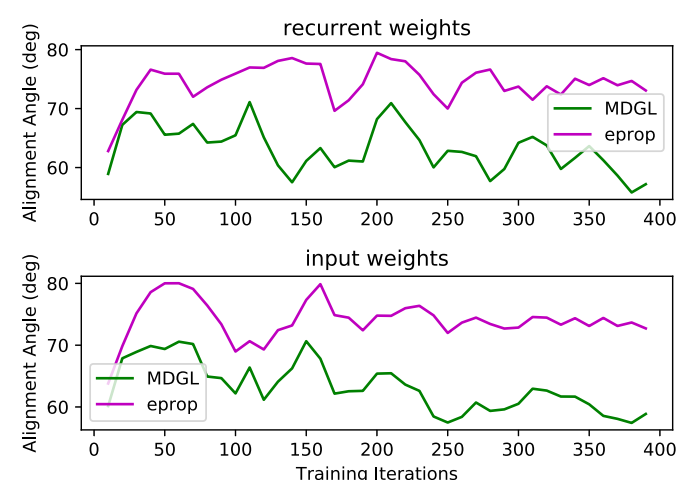

C
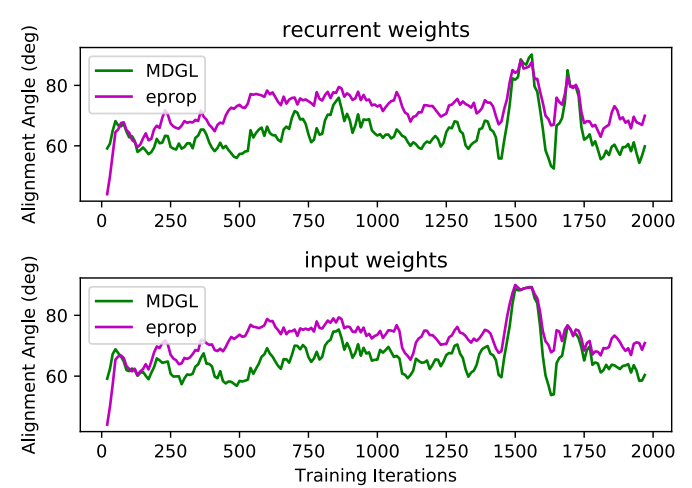

b
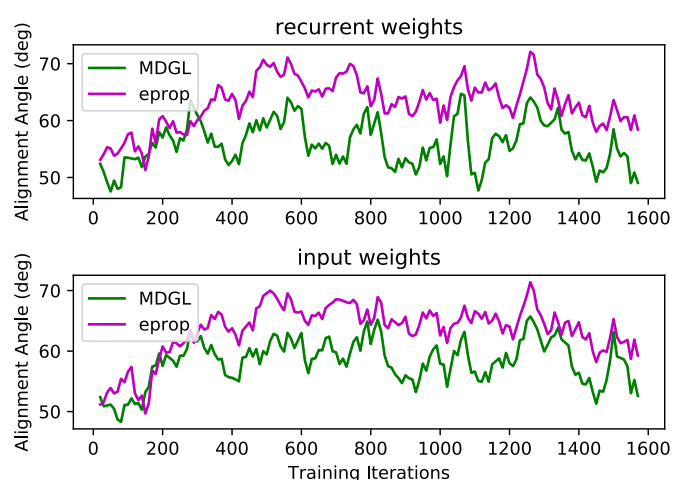

Figure S10: Alignment angle comparison shows that gradients approximated by MDGL is more similar (than e-prop) to the exact gradients. We quantify the similarity between approximated and exact gradients at each time point via the alignment angle, which describes the similarity in the direction of the two update vectors (Supplementary Note 3). This comparison is done for a) pattern generation, b) delayed match-to-sample and c) evidence accumulation tasks. All tasks were trained using MDGL. In each subfigure, alignment angles for recurrent (resp. input) weights over training iterations are shown in the top (resp. bottom) panel. 


\section{Supplementary Tables}

Table S1: Local modulatory terms of MDGL and TRTRL are significantly aligned

\begin{tabular}{ccccccc}
\hline \multirow{2}{*}{ Task } & \multicolumn{2}{c}{ Beginning } & \multicolumn{2}{c}{ Middle } & \multicolumn{2}{c}{ End of Training } \\
& Alignment $\left(^{\circ}\right)$ & Z-score & Alignment $\left(^{\circ}\right)$ & Z-score & Alignment $\left({ }^{\circ}\right)$ & Z-score \\
\hline Pattern Generation & 36 & -387 & 36 & -385 & 44 & -317 \\
Delayed Match to Sample & 22 & -114 & 48 & -74 & 32 & -99 \\
Evidence Accumulation & 50 & -67 & 44 & -81 & 55 & -59 \\
\hline
\end{tabular}

The alignment angles are computed between the local modulatory terms due to neuron-specific rather than type-specific gain (the vectorization of $2 \mathrm{D}$ matrices $\widehat{\Gamma_{p q}}=\sum_{t} \widehat{\Gamma_{p q, t}}$ of TRTRL in Eq. 3 and those due to the cell-type-specific approximation $\Gamma_{p q}=\sum_{t} \Gamma_{p q, t}$ of MDGL in Eq. 5 using E and I cell types. The angles listed here are for recurrent weight update, and similar values are observed for input weight updates. Here, beginning, middle and end of training refer to after 10, 100 and 500 training iterations, respectively, for the pattern generation task, and after 100, 500 and 2000 training iterations, respectively, for the delayed match to sample and evidence accumulation tasks. Z-scores were computed to show the significance of the difference of alignment from $90^{\circ}$; these scores suggest that TRTRL and MDGL are significantly aligned. Details for computing Z-scores and alignment angles are provided in Supplementary Note 3

Table S2: Local modulatory term of MDGL is not significantly aligned with the approximated gradient by e-prop

\begin{tabular}{ccccccc}
\hline \multirow{2}{*}{ Task } & \multicolumn{2}{c}{ Beginning } & \multicolumn{2}{c}{ Middle } & \multicolumn{2}{c}{ End of Training } \\
& Alignment $\left({ }^{\circ}\right)$ & Z-score & Alignment $\left(^{\circ}\right)$ & Z-score & Alignment $\left(^{\circ}\right)$ & Z-score \\
\hline Pattern Generation & 89 & -8.4 & 80 & -69 & 92 & 16 \\
Delayed Match to Sample & 94 & 5.3 & 88 & -2.3 & 92 & 2.2 \\
Evidence Accumulation & 91 & 1.2 & 91 & 1.4 & 89 & -1.2 \\
\hline
\end{tabular}

Similar to Table $\mid \mathrm{S} 1$ but the alignment angles are computed between the vectorization of $\Gamma_{p q}=\sum_{t} \Gamma_{p q, t}$ of MDGL in Eq. 5 (the local modulatory term using $\mathrm{E}$ and I cell types) and the approximated error gradient $\left.\frac{\mathrm{d} E}{\mathrm{~d} w_{p q}}\right|_{e-p r o p}$ of e-prop. Z-scores are computed to determine the significance of the difference of alignment from $90^{\circ}$ (which indicates gradients are not aligned). These scores show that the alignment angles are not significantly below $90^{\circ}$, indicating that cell-type-specific local modulatory signaling is offering additional information over and above e-prop. Formulae for Z-scores and alignment angle are provided in Supplementary Note 3

\section{Supplementary Note 1 - Online Learning for Leaky Output}

Consider a supervised learning task with loss $E=\sum_{k, t}\left(y_{k, t}^{*}-y_{k, t}\right)^{2}$ and leaky output $y_{k, t}=\kappa y_{k, t-1}+\sum_{j} w_{k j}^{\text {OUT }} z_{j, t}+$ $b_{k}^{\text {OUT }}$, we have the following partial derivative

$$
\frac{\partial E}{\partial z_{j, t}}=\sum_{k} w_{k j}^{O U T} \sum_{t^{\prime} \geq t}\left(y_{t^{\prime}, k}^{*}-y_{t^{\prime}, k}\right) \kappa^{t^{\prime}-t} .
$$

This seemingly provides an obstacle for online learning, because $\frac{\partial E}{\partial z_{j, t}}$ depends on future errors. However, changing the summation order [48] solves this problem and the following derivation can be generalized to the classification task with a simple replacement of $\left(y_{k, t}^{*}-y_{k, t}\right)$ by $\left(\pi_{k, t}^{*}-\pi_{k, t}\right)$ :

$$
\begin{aligned}
\left.\frac{\mathrm{d} E}{\mathrm{~d} w_{p q}}\right|_{e-p r o p} & =\sum_{t^{\prime}} \frac{\partial E}{\partial z_{p, t^{\prime}}} e_{p q}^{t^{\prime}} \\
& =\sum_{k, t^{\prime}} w_{k j}^{O U T} \sum_{t \geq t^{\prime}}\left(y_{k, t}^{*}-y_{k, t}\right) \kappa^{t-t^{\prime}} e_{p q}^{t^{\prime}} \\
& =\sum_{k, t} w_{k j}^{O U T}\left(y_{k, t}^{*}-y_{k, t}\right) \underbrace{\sum_{t^{\prime} \leq t} \kappa^{t-t^{\prime}} e_{p q}^{t^{\prime}}}_{\mathcal{F}_{\kappa}\left(e_{p q}^{t}\right)},
\end{aligned}
$$

where the order of summations was changed in the last line, and operator $\mathcal{F}_{\kappa}$ denotes low-pass filtering with $\mathcal{F}_{\kappa}\left(x_{t}\right)=\kappa \mathcal{F}_{\kappa}\left(x_{t-1}\right)+x_{t}$. In our actual implementation, we used an exponential smoothing with $\mathcal{F}_{\kappa}\left(x_{t}\right)=$ $\kappa \mathcal{F}_{\kappa}\left(x_{t-1}\right)+(1-\kappa) * x_{t}$, but dropped the factor $(1-\kappa)$ in writing for readability. 
We also apply the change of summation order trick to the cell-type-specific modulatory signal $\Gamma_{p q, t}$ and assume that activity of neuron $j$ is not correlated with the eligibility trace of synapse $p q$ :

$$
\begin{aligned}
\sum_{t^{\prime}} \Gamma_{p q, t^{\prime}} & =\sum_{t^{\prime}, j \neq p} \frac{\partial E}{\partial z_{j, t^{\prime}}} h_{j, t^{\prime}} w_{\alpha \beta} e_{p q}^{t^{\prime}-1} \\
& =\sum_{k, t^{\prime}, j \neq p} w_{k j}^{O U T} \sum_{t \geq t^{\prime}}\left(y_{k, t}^{*}-y_{k, t}\right) \kappa^{t-t^{\prime}} h_{j, t^{\prime}} w_{\alpha \beta} e_{p q}^{t^{\prime}-1} \\
& \stackrel{(a)}{=} \sum_{t} \sum_{j \neq p} \sum_{k}\left(y_{k, t}^{*}-y_{k, t}\right) w_{k j}^{O U T} w_{\alpha \beta} \underbrace{\left[\kappa^{t-t^{\prime}} h_{j, t} e_{p q}^{t-1}\right]}_{\approx\left(t-t^{\prime}+1\right) \mathbb{E}_{t^{\prime} \leq t} \sum_{t^{\prime} \leq t} \kappa^{t-t^{\prime}} h_{j, t^{\prime}} e_{p q}^{t^{\prime}-1}} \\
& \stackrel{(b)}{\approx} \sum_{t} \sum_{j \neq p} \sum_{k}\left(y_{k, t}^{*}-y_{k, t}\right) w_{k j}^{O U T} w_{\alpha \beta} \mathbb{E}_{t^{\prime} \leq t}\left[h_{j, t}\right] \underbrace{\left(t-t^{\prime}+1\right) \mathbb{E}_{t^{\prime} \leq t}\left[\kappa^{t-t^{\prime}} e_{p q}^{t-1}\right]}_{=\mathcal{F}_{\kappa}\left(e_{p q}^{t-1}\right)} \\
& =\sum_{t} \sum_{j \neq p} \sum_{k}\left(y_{k, t}^{*}-y_{k, t}\right) w_{k j}^{O U T} w_{\alpha \beta} \underbrace{\mathbb{E}_{t^{\prime} \leq t}\left[h_{j, t}\right]}_{\approx \mathcal{F}_{\kappa}\left(h_{j, t}\right)} \mathcal{F}_{\kappa}\left(e_{p q}^{t-1}\right) \\
& \stackrel{(c)}{\approx} \sum_{t} \sum_{j \neq p} \sum_{k}\left(y_{k, t}^{*}-y_{k, t}\right) w_{k j}^{O U T} w_{\alpha \beta} \mathcal{F}_{\kappa}\left(h_{j, t}\right) \mathcal{F}_{\kappa}\left(e_{p q}^{t-1}\right) \\
& =\sum_{t} \sum_{j \neq p}[\underbrace{}_{\left.:=\sum_{k}\left(y_{k, t}^{*}-y_{k, t}\right) w_{k j}^{O U T} \mathcal{F}_{\kappa}\left(h_{j, t}\right)\right]} w_{\alpha \beta} \mathcal{F}_{\kappa}\left(e_{p q}^{t-1}\right) \\
& \stackrel{(d)}{=} \sum_{t} \mathcal{F}_{\kappa}\left(e_{p q}^{t-1}\right) \sum_{\alpha \in C} w_{\alpha \beta} \sum_{j \in \alpha} \bar{a}_{j, t},
\end{aligned}
$$

where (a) changes the summation order; $(b)$ assumes uncorrelatedness between activity $h_{j, t}$ and $\kappa^{t-t^{\prime}} e_{p q}^{t-1}$ such that $\mathbb{E}_{t^{\prime} \leq t}\left[\kappa^{t-t^{\prime}} h_{j, t} e_{p q}^{t-1}\right] \approx \mathbb{E}_{t^{\prime} \leq t}\left[h_{j, t}\right] \mathbb{E}_{t^{\prime} \leq t}\left[\kappa^{t-t^{\prime}} e_{p q}^{t-1}\right] ;(c)$ approximates the temporal average of $h_{j, t}$ using an exponential filter $\mathbb{E}_{t^{\prime} \leq t}\left[h_{j, t}\right] \approx \mathcal{F}_{\kappa}\left(h_{j, t}\right) ;(d)$ is a simple change of summation order. We test the validity of above approximation in Figure $\mathrm{S} 3$ and observe no significant performance degradation due to this approximation.

\section{Supplementary Note 2 - Detailed Breakdown of MDGL's Components}

In the main text, we stated that our MDGL learning rule combines the eligibility trace with both top-down learning signals and cell-type-specific weighted summation of secreted, diffuse modulators. We so far only expressed these components as derivatives. With the derivation of the online implementation for MDGL in Eq. S3, we are now ready to provide the detailed expressions for each of these components. Combining Eq. S3 with Eq. 5 and rearranging the summation order gives the following component breakdown for our online approximation to MDGL:

$$
\frac{\widehat{\mathrm{d} E}}{\mathrm{~d} w_{p q}} \approx\left[\sum_{k}\left(y_{t-1, k}^{*}-y_{t-1, k}\right)(w_{k p}^{O U T}+\underbrace{\sum_{\alpha \in C} w_{\alpha \beta} \sum_{j \in \alpha} w_{k j}^{O U T} \mathcal{F}_{\kappa}\left(h_{j, t-1}\right)}_{\text {Our addition }})\right] \mathcal{F}_{\kappa}\left(e_{p q, t-1}\right) .
$$

The non-neuron-specific error signal $\left(y_{t, k}^{*}-y_{t, k}\right)$ is passed to cells through neuron-specific feedback weights $w_{k j}^{O U T}$, thereby forming neuron-specific learning signal at the receiving end $L_{j, t}=\sum_{k} w_{k j}^{O U T}\left(y_{t, k}^{*}-y_{t, k}\right)$. Thus, loosening this neuron-specificity of learning signal can be achieved through approximations to the feedback weights, such as replacing them with random weights [64] or cell-type-specific gains as in Eq. 4. Upon receipt, neuron $j$ multiplies $L_{j, t}$ with $\mathcal{F}_{\kappa}\left(h_{j, t}\right)$, its low-pass filtered activity, and sends the packaged signal $a_{j, t}=L_{j, t} \mathcal{F}_{\kappa}\left(h_{j, t}\right)$. In updating $w_{p q}$, our addition allows postsynaptic cell $p$ to collect information regarding the activities and learning signals of other cells through cell-type-specific gain $w_{\alpha \beta}$, and combine the received modulatory input with its low-pass filtered eligibility trace. 


\section{Supplementary Note 3 - Analysis and Simulation Details}

Throughout this study, the alignment angle $\theta$ between two vectors, a and b, was computed by $\theta=a \cos \left(\left\|a^{T} b\right\| /\|a\|\|b\|\right)$. The alignment between two $2 \mathrm{D}$ matrices was computed by flattening the matrices into vectors. To obtain the significance of alignment in Tables S1 and S2, we randomly shuffled the matrices, calculated the resulting alignment angle and repeated for 1000 times to obtain an empirical distribution of alignment angles. The mean $\mu$ and standard deviation $\sigma$ were computed from the distribution to report the Z-score $=\frac{\theta-\mu}{\sigma}$.

For spectral analysis, we first performed root mean square normalization on the signal and then computed the power spectral density using Welch's method [99]. We then found the $3 \mathrm{~dB}$ frequency by identifying the maximum frequency at which the power is halved from the peak power.

For the pattern generation task in Figure 4, our network consisted of 400 LIF neurons. All neurons had a membrane time constant of $\tau_{m}=30 \mathrm{~ms}$, a baseline threshold of $v_{\text {th }}=0.01$ and a refractory period of $2 \mathrm{~ms}$. Input to this network was provided by 100 Poisson spiking neurons with a rate of $10 \mathrm{~Hz}$. The fixed target signal had a duration of $2000 \mathrm{~ms}$ and given by the sum of five sinusoids, with fixed frequencies of $0.5 \mathrm{~Hz}, 1 \mathrm{~Hz}, 2 \mathrm{~Hz}, 3 \mathrm{~Hz}$ and $4 \mathrm{~Hz}$. For learning, we used mean squared loss function and for visualization, we used normalized mean squared error NMSE $=\frac{\sum_{k, t}\left(y_{k, t}^{*}-y_{k, t}\right)^{2}}{\sum_{k, t}\left(y_{k, t}^{*}\right)^{2}}$ for zero-mean target output $y_{k, t}^{*}$. All weight updates were implemented using Adam with default parameters $[100]$ and a learning rate of $1 \times 10^{-3}$. In addition, we applied firing rate regularization with $c_{\text {reg }}=10$ and $f^{\text {target }}=10 \mathrm{~Hz}$.

For the delayed match to sample task in Figure 5, our network consisted of 50 LIF neurons and 50 ALIF neurons (100 LIF neurons and 80 ALIF neurons for the alternative setup in Figure S5). All neurons had a membrane time constant of $\tau_{m}=20 \mathrm{~ms}$, a baseline threshold of $v_{\text {th }}=0.01$ and a refractory period of $5 \mathrm{~ms}$. The time constant of threshold adaptation was set to $\tau_{b}=1400 \mathrm{~ms}$, and its impact on the threshold was set to $\beta=1.8$. Input to this network was provided by three populations, as illustrated in Figure 5B. The first (resp. second) population consisted of 20 units and produced Poisson spike trains with a rate of $40 \mathrm{~Hz}$ when the first (resp. second) cue takes a value of 1 , otherwise it stays quiescent. The last input population of 10 units produced Poisson spike trans of $10 \mathrm{~Hz}$ throughout the trial in order to prevent the network from being quiescent during the delay. For the alternative setup in Figure S5, the first (resp. second) input population produced Poisson spike trains with a rate of $40 \mathrm{~Hz}$ when cue 1 (resp. cue 2) is presented, otherwise it fires at 10Hz. For learning, we used cross-entropy loss function and the target corresponding to the correct output was given at the end of the trial. As done in the evidence accumulation task, a weight update was applied once every 64 trials and the gradients were accumulated during those trials additively. All weight updates were implemented using Adam with default parameters 100 and a learning rate of $2.5 \times 10^{-3}$. In addition, we applied firing rate regularization with $c_{\text {reg }}=0.1$ and $f^{\text {target }}=10 \mathrm{~Hz}$.

For the evidence accumulation task in Figure 6, our network consisted of 50 LIF neurons and 50 ALIF neurons. All neurons had a membrane time constant of $\tau_{m}=20 \mathrm{~ms}$, a baseline threshold of $v_{\text {th }}=0.01$ and a refractory period of $5 \mathrm{~ms}$. The time constant of threshold adaptation was set to $\tau_{b}=2000 \mathrm{~ms}$, and its impact on the threshold was set to $\beta=1.8$. Input to this network was provided by four populations of 10 neurons each, as illustrated in Figure $6 \mathrm{~B}$. The first (resp. the second) population produced Poisson spike trains with a rate of $40 \mathrm{~Hz}$ when a cue was presented on the left (resp. right) side of the track. The third input population spiked randomly through the decision period with a firing rate of $40 \mathrm{~Hz}$ and was silent otherwise. The last input population produced Poisson spike trains with a rate of $10 \mathrm{~Hz}$ throughout the trial in order to prevent the network from being quiescent during the delay. For learning, we used the cross-entropy loss function and the target corresponding to the correct output was given at the end of the trial. A weight update was applied once every 64 trials and the gradients were accumulated during those trials additively. All weight updates were implemented using Adam with default parameters 100 and a learning rate of $2.5 \times 10^{-3}$. In addition, we applied firing rate regularization with $c_{\text {reg }}=0.1$ and $f^{\text {target }}=10 \mathrm{~Hz}$. For all simulations, we used a time step of $1 \mathrm{~ms}$, as done in 48 . We also assumed a synaptic delay of $1 \mathrm{~ms}$ for all synapses. 